\title{
The Multi-Circuit Neuronal Hyperexcitability Hypothesis of Psychiatric Disorders
}

\author{
Michael R. Binder ${ }^{1,2}$ \\ ${ }^{1}$ North Shore University Health System Highland Park Hospital, Highland Park, USA \\ ${ }^{2}$ Pritzker School of Medicine, The University of Chicago, Chicago, USA
}

Email address:

mbinder@drmichaelbinder.com

\section{To cite this article:}

Michael R. Binder. The Multi-Circuit Neuronal Hyperexcitability Hypothesis of Psychiatric Disorders. American Journal of Clinical and Experimental Medicine. Vol. 7, No. 1, 2019, pp. 12-30. doi: 10.11648/j.ajcem.20190701.13

Received: January 15, 2019; Accepted: March 12, 2019; Published: March 30, 2019

\begin{abstract}
After nearly a century of intensive research and clinical investigation, the pathophysiology of psychiatric disorders remains an enigma. Short of a clear understanding of how psychiatric symptoms are produced, the various cognitive, emotional, and behavior patterns that characterize psychiatric disorders continue to be grouped into syndromes and treated accordingly. The weakness of this approach is that the treatment is administered without a clear understanding of what pathological process is being treated. Moreover, the symptoms of most psychiatric disorders are frequently changing and melding into one another. This leads to diagnostic confusion, medication stacking, and poor treatment outcomes, all of which erode patient trust and perpetuate the stigma of mental illness. In this seminal report, a new hypothesis on the pathophysiology of psychiatric disorders will be presented based on multidisciplinary evidence that nearly all psychiatric disorders and their functional comorbidities are rooted in a single, shared, neurophysiological abnormality. The report will then trace that abnormality to its molecular roots and introduce a new paradigm through which the many faces of mental illness can be understood and uniformly treated. The Multi-Circuit Neuronal Hyperexcitability hypothesis of psychiatric disorders posits that an inherent hyperexcitability of the neurological system is at the root of mental illness and provides a precise, functionallyspecific framework that eliminates diagnostic confusion, informs a unified treatment approach, and helps remove the long-held stigma of mental illness.
\end{abstract}

Keywords: Hyperactive Brain, Central Sensitivity, Psychophysiology of Psychiatric Disorders, Brain Circuits in Psychiatric Disorders, Neural Circuits in Psychiatric Disorders, Genetics of Psychiatric Disorders, Temperament and Psychiatric Disorders, Neuromodulators

\section{Introduction}

Throughout human history, emotional and behavioral disorders have been grossly misunderstood. During ancient times, the mentally ill were thought to be demon-possessed, and behavioral disorders were assumed to be a form of punishment for sins committed. With few exceptions, these beliefs prevailed through the Middle Ages, and it was not until the beginning of the last century that modern ideas about the causation and treatment of mental illness began to emerge. Today, despite great strides in the field of neuroscience, the pathophysiology of psychiatric disorders remains poorly understood.

Consequently, the various cognitive, emotional, and behavioral patterns that characterize psychiatric disorders continue to be grouped into different syndromes, labeled as separate illnesses, and treated accordingly. The weakness of this approach is that the treatment is administered without a clear understanding of what pathological process is being treated. Moreover, the symptoms of most psychiatric disorders are frequently changing and melding into one another. This leads to diagnostic confusion, medication stacking, and erosion of patient trust in the mental healthcare system.

In an effort to address these issues, various theories about the causality of mental illness have emerged, but none have fully integrated the inner workings of the human mind with the functional dynamics of the brain to provide a precise, psychophysiological framework through which psychiatric 
disorders can be understood. Consequently, there continue to be conflicting views about how to best treat mental illness, with some advocating psychotherapeutic interventions, others, biological interventions, and still others, natural interventions such as diet, exercise, and meditation. A better understanding of the psychophysiology of psychiatric disorders would help resolve these differences, simplify diagnostics, and streamline treatment. This in turn would increase patient trust, improve treatment outcomes, and reduce the cost of mental health care.

In this seminal report, a new hypothesis on the pathophysiology of psychiatric disorders will be presented based on biological, observational, pharmacological, neuropsychiatric, behavioral, medical, psychophysiological, experimental, radiological, and explanatory evidence that nearly all psychiatric disorders and their functional comorbidities are rooted in a single, shared, neurophysiological abnormality. After presenting the evidence for the hypothesis, the discussion will turn to the striking connection between the top candidate genes for the major psychiatric disorders and the proposed pathophysiology of those disorders to provide a cohesive theory that dissects psychiatric disorders down to their molecular roots and provides a functionally-specific framework through which nearly all psychiatric disorders and their functional comorbidities can be understood and uniformly treated.

\section{Development of the Hypothesis}

A proper understanding of psychiatric disorders logically begins with a discussion of brain function as it relates to the mind. The brain is a biological computer that has a specific circuit (or set of circuits) that corresponds to every conceivable thought, feeling, and experience [1-4]. There are circuits for love, circuits for joy, circuits for fear, circuits for anger; indeed, there are circuits for every conceivable thought and emotion. Like tapping keys on a computer, the appropriate circuit is activated each time one engages in a particular cognitive-emotional function [5]. The reverse process also occurs; each time a specific circuit is activated, one thinks, feels, or experiences whatever it is that that circuit corresponds to [6].

Given that every thought and feeling both affects and is affected by the neurological system of the brain, it would not be unreasonable to think that if the electrical activity in any one of the brain's circuits became dominant, the associated thought or feeling would likewise become dominant. Normally, such circuit-specific imbalances would be mild and transient, persisting only as long as a particular emotion or train of thought were occurring. However, pathological circuit-specific imbalances; that is, ones that were unusually large or persistent, would be expected to involve unusually intense or abnormally prolonged thoughts and feelings. For example, if depressive circuits became persistently hyperactive, one would experience persistent thoughts and feelings of depression. If anxiety circuits became persistently hyperactive, one would experience persistent feelings of anxiety. If circuits for pleasure became persistently hyperactive, one would experience persistent feelings of euphoria. In other words, the persistence of pathological levels of hyperactivity in specific circuits would create the symptoms that have been grouped into the various psychiatric disorders.

In thinking about the underlying cause of these pathological states, it is important to note that none of them are abnormal in nature; they are merely abnormal in degree. At the same time, it is well-known that the most ubiquitous trigger of psychiatric symptoms is cognitive-emotional stress, which has a graded excitatory effect on the neurological system $[7,8]$. This leads to the hypothesis that persons who are vulnerable to mental illness have a neurological system that is pathologically hyperexcitable. An inherent vulnerability of this type would not be expected to cause any significant symptoms if the stress level were low; but if the stress level were to rise, the modest circuit-specific imbalances that were driven by normal cognitive and emotional processing would become overamplified. This would further increase the perceived level of stress, which in turn would further increase the magnitude and duration of the circuit-specific imbalances. This vicious cycle is what the Multi-Circuit Neuronal Hyperexcitability (MCNH) hypothesis proposes to be the cause of the escalating psychiatric symptoms that some persons experience in the face of severe or persistent cognitive-emotional distress.

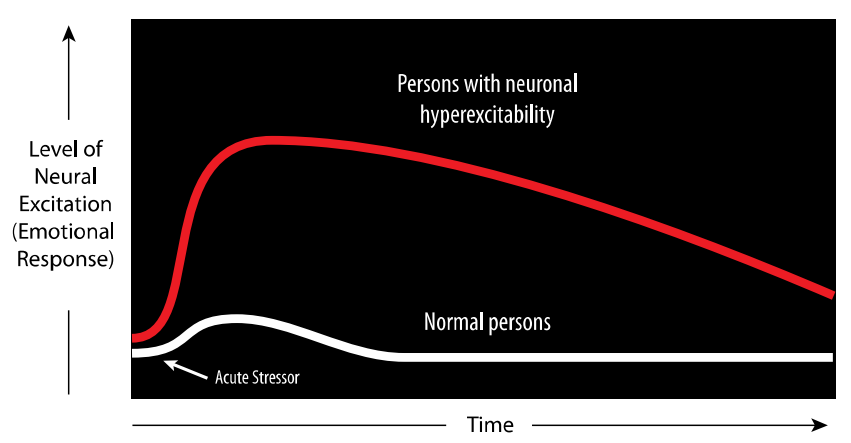

Figure 1. Conceptual illustration of the stress response in a person with neuronal hyperexcitability in comparison to a normal response. Note the heightened intensity and duration of the neuronal excitation and, correspondingly, the emotional response in the person with hyperexcitable neurons.

What theoretically underlies the abnormal stress response is a hyperexcitability of the neurological system; the neurons simply overreact. The response is also prolonged both because the neurons have a compromised ability to selfregulate and because they are repeatedly stimulated as the individual processes the distressing thoughts and feelings (Figure 1). The abnormality becomes even more pronounced when multiple stressors occur in rapid succession (Figure 2). In addition, the hyperactivity in the system accelerates a phenomenon called primed burst potentiation, a physiological form of kindling in which circuits that are frequently used become increasingly responsive over time [9]. While this adaptive process facilitates learning, it also 
tends to increase the intensity and duration of the stress response, particularly if the neurons are inherently hyperexcitable.

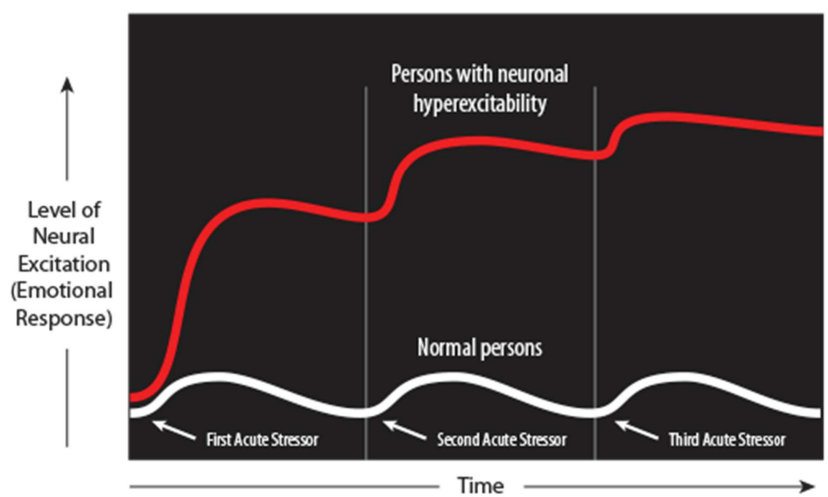

Figure 2. Conceptual illustration of the stress response in a person with neuronal hyperexcitability in comparison to a normal response when repeated stressors occur in short succession. Note how high the level of neuronal excitation and, correspondingly, the intensity of the emotional response can rise if there is not enough time to recover from each stressful event.

Thus, the MCNH hypothesis provides a precise psychophysiologic mechanism for the long-held diathesisstress model of mental illness and directly explains the almost universal benefits of stress-reduction, adequate sleep, proper diet, regular exercise, mindful meditation, spirituality, and psychotherapy in the treatment and prevention of mental illness. The hypothesis is further supported by the following ten multidisciplinary forms of evidence and affirmed by the genetic findings that will be presented in sections 4 and 5 .

\section{The Multidisciplinary Evidence}

\subsection{Biological Evidence}

Biological evidence for the neuronal hyperexcitability hypothesis comes from the study of epilepsy and the close relationship between seizure disorders and psychiatric disorders. The idea that the two disorder-types are closely related dates back to around 400 B. C., when Hippocrates wrote, "Melancholics ordinarily become epileptics, and epileptics, melancholics: what determines the preference is the direction the malady takes; if it bears upon the body, epilepsy, if upon the intelligence, melancholy." Seizures occur when large populations of neurons begin to fire hypersynchronously [10]. Because the risk of hypersynchrony increases as the level of excitation in the brain increases, the catalyst for a seizure is any condition or chemical that increases the excitability of the neurological system, such as emotional stress, an inflammatory process, a metabolic disturbance, or a stimulant-type drug. An important clue to the pathophysiology of psychiatric disorders is that the same conditions and chemicals that increase the potential for seizures also increase the potential for psychiatric symptoms, and the same conditions and chemicals that decrease the potential for seizures also decrease the potential for psychiatric symptoms (Table 1). That tells us that neuronal hyperexcitability is a catalyst for psychiatric symptoms.

Table 1. Comparison of the Effects of Various Conditions and Chemicals on Seizure and Psychiatric Symptom Potential.

\begin{tabular}{|c|c|c|}
\hline CONDITIONS and CHEMICALS & $\begin{array}{l}\text { SEIZURE } \\
\text { POTENTIAL }\end{array}$ & $\begin{array}{l}\text { PSYCHIATRIC SYMPTOM } \\
\text { POTENTIAL }\end{array}$ \\
\hline Cognitive-emotional stress [11] & $\uparrow$ & $\uparrow$ \\
\hline Sleep deprivation [12] & $\uparrow$ & $\uparrow$ \\
\hline Psychostimulants (caffeine, methamphetamine, phencyclidine) $[13,14]$ & $\uparrow$ & $\uparrow$ \\
\hline Sedative withdrawal (benzodiazepines, barbiturates, hypnotics, alcohol) & $\uparrow$ & $\uparrow$ \\
\hline Inflammation [15-17] & $\uparrow$ & $\uparrow$ \\
\hline Dietary sugar (especially refined sugar) $[18,19]$ & $\uparrow$ & $\uparrow$ \\
\hline Experimentally induced kindling of the brain $[22,23]$ & $\uparrow$ & $\uparrow$ \\
\hline Stress reduction [11] & $\downarrow$ & $\downarrow$ \\
\hline Progesterone [24-26] & $\downarrow$ & $\downarrow$ \\
\hline Anticonvulsant drugs & $\downarrow$ & $\downarrow$ \\
\hline Sedatives (benzodiazepines, barbiturates, hypnotics, alcohol, some cannabinoids) [27-29] & $\downarrow$ & $\downarrow$ \\
\hline Centrally-acting alpha-2 agonists (clonidine, guanfacine) $[30,31]$ & $\downarrow$ & $\downarrow$ \\
\hline Acamprosate [32-36] & $\downarrow$ & $\downarrow$ \\
\hline Riluzole $[37,38]$ & $\downarrow$ & $\downarrow$ \\
\hline Magnesium $[40,41]$ & $\downarrow$ & $\downarrow$ \\
\hline Taurine $[42,43]$ & $\downarrow$ & $\downarrow$ \\
\hline Ketogenic diet $[18,19]$ & $\downarrow$ & $\downarrow$ \\
\hline Omega-3 fatty acids $[44,45]$ & $\downarrow$ & $\downarrow$ \\
\hline Plant-based oils and alkaloids [46-51] & $\downarrow$ & $\downarrow$ \\
\hline Electroconvulsive Therapy (ECT) $[52,53]$ & $\downarrow$ & $\downarrow$ \\
\hline Vagus Nerve Stimulation (VNS) $[54,55]$ & $\downarrow$ & $\downarrow$ \\
\hline Deep Bran Stimulation (DBS) $[56,57]$ & $\downarrow$ & $\downarrow$ \\
\hline
\end{tabular}

+ Itemized comparison showing that the same conditions and chemicals that increase the potential for seizures (upper section) also increase the potential for psychiatric symptoms, and the same conditions and chemicals that decrease the potential for seizures (lower section) also decrease the potential for psychiatric symptoms. 
Referring again to Table 1, note that seizures themselves, which can be clinically indistinguishable from psychiatric symptoms [58-61], can, paradoxically, be used (in the form of ECT) to treat both seizures and psychiatric symptoms [52, 53]. Although the mechanism by which ECT relieves psychiatric symptoms remains unclear, it is evident that clinical improvement occurs not during the seizure but in the aftermath of the seizure. It is now recognized that seizures are brought to a halt by a host of neuroinhibitory changes that occur in response to the seizures themselves. Known mechanisms include glutamate depletion, GABAergic recurrent inhibition, membrane shunting, depletion of energy stores, loss of ionic gradients, endogenous neuromodulator effects, and regulatory input from various brain regions [62]. That a remission of depression and other psychiatric symptoms occurs in conjunction with this robust inhibitory activity is further evidence that psychiatric symptoms are rooted in neuronal hyperexcitability.

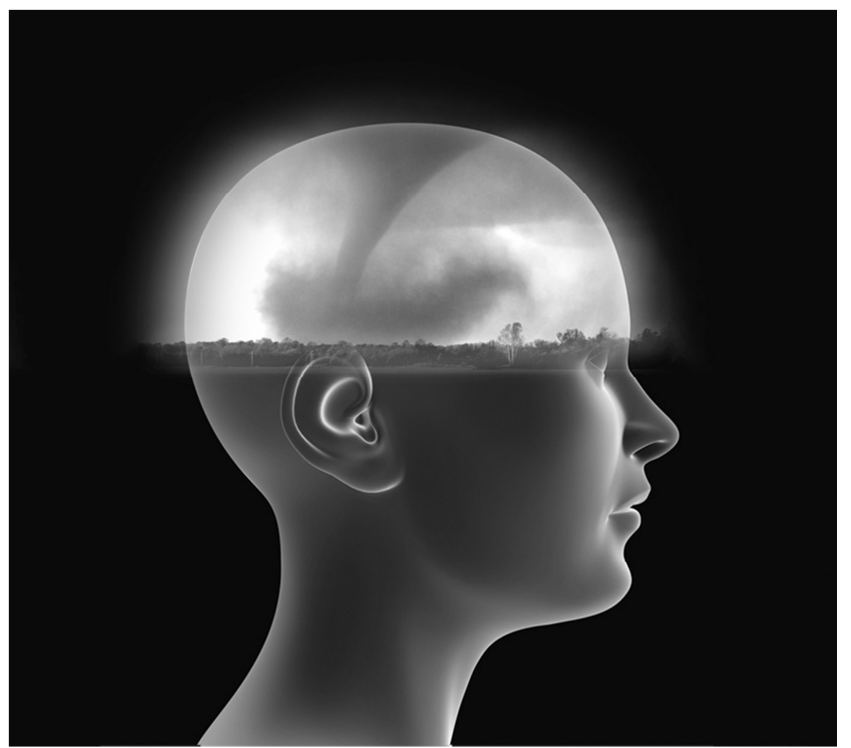

Figure 3. The tornado in the illustration represents the locus of neuronal hyperactivity as it migrates from one circuit loop to another to stir up various psychiatric symptoms in the hyperexcitable brain.

Another feature of seizure disorders that provides a clue to the pathophysiology of psychiatric disorders is the migration of symptoms. From the study of epilepsy, it is known that focal areas of hyperactivity do not necessarily remain focal; instead, they tend to migrate from one group of neurons to another, and from one group of circuits to another like a wandering tornado (Figure 3). This process is what underlies the classic "Jacksonian march" of convulsive activity. A similar migration of symptoms occurs in psychiatric disorders. What theoretically allows symptoms such as anxiety, depression, and irritability to oscillate back and forth and morph into one another is that the circuit-specific hyperactivity that is driving the symptoms fuels hyperactivity in various circuit loops that would be resistant to such aberrant induction were they not themselves hyperexcitable (Figure 4).



Aberrant circuit activation

Figure 4. Schematic illustration of one of the means by which one hyperactive circuit can aberrantly fuel hyperactivity in another circuit to cause manic-depressive switching. In this example, the depressive circuit loop and the manic circuit loop inappropriately excite each other. This is more apt to occur in persons with neuronal hyperexcitability both because the neurological system tends to be more hyperactive and because collateral circuits, which themselves are hyperexcitable, are more easily brought to threshold by feeder circuits.

Of course, the duration of a psychiatric episode is much longer than that of an epileptic seizure. That is theoretically because the number of neurons involved in the induction of psychiatric symptoms is too small and unsynchronized to stimulate the neuroinhibitory changes that bring seizures to a halt (that is, unless it is bolstered by ECT). It is also too small and unsynchronized to induce enough magnetic field strength to cause the rapid migration of symptoms, disruption of consciousness, and electroencephalographic changes that characterize seizure activity. Nonetheless, the fact that psychiatric symptoms, like convulsive symptoms, tend to migrate is another line of evidence that they are driven by neuronal hyperactivity.

Hormonal evidence that psychiatric disorders are rooted in neuronal hyperexcitability comes from the study of women with the hormonally-related disorders of premenstrual syndrome, premenstrual dysphoric disorder, and postpartum depression. These disorders, which are characterized by symptoms that are found in a variety of psychiatric disorders and their functional comorbidities, are well-known to be hormonally-related. What is not clear is how they are hormonally-related [63]. However, a careful analysis of the hormonal changes that occur during the menstrual cycle clearly indicates that premenstrual symptoms begin not when estrogen falls (at mid-cycle) but when progesterone falls (about 10 days later) (Figure 5). Note that the progesterone level also falls at parturition, which is the other time at which women are more vulnerable to developing psychiatric symptoms (in the form of postpartum depression). Conversely, most women report an increasing sense of wellbeing as the progesterone level climbs during pregnancy (Figure 6). Unlike estrogen, progesterone is an anticonvulsant [24-26]. This provides hormonal evidence that psychiatric disorders and their functional comobidities are rooted in neuronal hyperexcitability. 




Figure 5. Illustration of the close temporal relationship between the fall in progesterone level and the start of premenstrual psychiatric symptoms (shaded area). Note that premenstrual symptoms do not begin when estrogen falls (at mid-cycle) but when progesterone falls (around day 22). For reference, when the menstrual cycle is abolished by the progesterone receptor antagonist mifepristone, the psychiatric symptoms still occur [64]. This teases out the effect of progesterone from the other hormonal changes involved in the menstrual cycle.

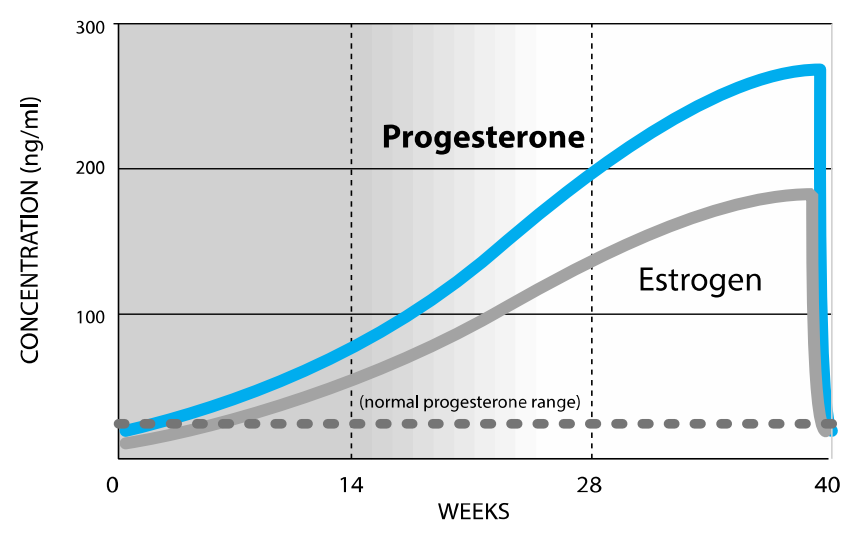

Figure 6. Illustration of the gradual remission of pre-pregnancy psychiatric symptoms (loss of dark shading) as the progesterone level climbs during pregnancy.

\subsection{Observational Evidence}

In obsessive-compulsive disorder (OCD), the persistence of activity in cortico-striato-thalamo-cortical (CSTC) circuit loops $[65,66]$ can readily be explained by the inability of hyperexcitable neurons to shut off. The idea that OCD is rooted in neuronal hyperexcitability would also explain why a sizable number of patients who are diagnosed with a psychiatric disorder other than OCD will acknowledge having $\mathrm{OC}$ tendencies. Moreover, these tendencies tend to become more pronounced during periods of high stress, when the overall level of excitation in the brain is higher and, thus, more likely to fuel hyperactivity in CSTC circuit loops. The reverse is also observed; persons with $\mathrm{OCD}$, in addition to experiencing a worsening of their OC symptoms, tend to develop other psychiatric symptoms during periods of high stress. In many cases, OC symptoms will alternate with other psychiatric symptoms over the course of an individual's lifetime. Though these other symptoms have heretofore been thought to represent different pathological processes, recent data suggest that they may actually be different manifestations of the same pathological process. In a 2018 study of nearly six million children and adults with a history of mental illness, McGrath et al [67] found that psychiatric comorbidity was more widespread than previously thought and not restricted to closely related psychiatric disorders. Specifically, various different disorders, ranging from mood and attentional disorders to schizophrenia and personality disorders, frequently manifested in the same person. What's more, the comorbid disorders tended to manifest within the same six-month period and were so highly bidirectional that chance alone could dictate which disorder would manifest first. From the perspective of the MCNH hypothesis, the specific symptoms that develop and the severity of those symptoms would depend upon which specific circuits were hyperactive and the extent to which they were hyperactive at any point in time.

Another psychiatric phenomenon that can readily be explained by the MCNH hypothesis is the symptom of psychosis. According to the MCNH hypothesis, psychotic symptoms develop when the level of electrical activity in the sensory processing system of the brain becomes as high as the level of activity that would normally be driven by input from the body's sensory organs. For example, aberrant discharges from neurons in the auditory processing system would cause the patient to think that the auditory nerve were being stimulated. This would lead to the false perception that sound was coming from the environment. Aberrant discharges from neurons in the visual processing system would lead to visual hallucinations, etc... Although such aberrant signaling could potentially occur in anyone, it would be more likely to occur in those with hyperexcitable neurons. This conceptualization is supported by a recent study that found that auditory hallucinations in schizophrenia were exaggerated versions of perceptual distortions that are not uncommonly experienced by persons who do not have schizophrenia [68]. The researchers found that the perceptual distortions were more pronounced in those participants whose neurons were releasing more dopamine, a neurotransmitter that helps modulate the processing of auditory signals [69]. From the perspective of the MCNH hypothesis, the excess dopamine release would be the result of hyperactivity in dopaminergic circuitry. Similarly, other forms of psychosis, such as paranoia and delusional thinking, would occur when the intensity of internally-generated, circuit-specific signaling began to approach the intensity of signaling that would normally be driven by the higher processing of visual, olfactory, tactile, and other sensory input. In other words, the hyperexcitable brain could amplify purely internal processes to the point that the mind, believing that the impetus is coming from the environmental, begins to weave the content into narratives to explain what it believes to reflect external reality. The risk of such aberrant signaling would be increased by intrapsychic stress, stimulant-type drugs, or any factor that increases excitation in the brain, thus explaining why psychotic symptoms are more likely to develop under such circumstances.

A related phenomenon that can likewise be explained by 
the MCNH hypothesis is the odd separation or "schism" between thoughts and feelings that define the term "schizophrenia." What theoretically causes this type of inappropriate affect is that cognitive functions that would normally activate the corresponding emotional circuitry are unable to do so because various hotspots of neural activity are competing for dominance [70]. As a result, the patient's emotions, rather than being dictated by the thought content, are dictated by inappropriate firing in limbic circuitry. It is also possible that the thought content, rather than being dictated by the emotions, could be dictated by inappropriate firing in cognitive circuitry. In theory, the willful intentions of the individual could be completely usurped by this intensive, spontaneous, electrical activity. Such chaotic brain signaling would be more likely to occur in patients with very high levels of neuronal excitability, such as those with schizophrenia, schizoaffective disorder, and other severe mental disorders. That such patients have exceptionally high levels of neuronal excitability is corroborated by the elevated risk of seizures that they have in comparison to those with less debilitating psychiatric disorders [71-73].

\subsection{Pharmacological Evidence}

Since the middle of the nineteenth century, when Sir Charles Locock first noted the beneficial effects of potassium bromide in the treatment of "hysterical epilepsy" [74], anticonvulsants and various tranquilizing drugs, which include barbiturates, benzodiazepines, non-benzodiazepine anticonvulsants, major tranquilizers, atypical antipsychotics, and sedative hypnotics, have been the mainstay in biological psychiatry. From the perspective of the MCNH hypothesis, these drugs exert their therapeutic effects by blocking or reducing neurotransmission in those circuits that are symptomatically hyperactive. In some cases, the hyperactivity may include dopaminergic circuits (leading to auditory hallucinations) and motor circuits (leading to agitation), thus explaining why a combination of an antipsychotic drug, such as haloperidol, and an anticonvulsant drug, such as lorazepam, can be so effective in the acute management of agitated psychotic patients. Another line of support for the idea that most psychotherapeutic drugs work by reducing neuronal hyperactivity is the added benefit of rapid stress reduction in severely ill patients. Traditionally accomplished through emergency psychiatric hospitalization, a rapid reduction in stress quickly reduces mental tension, which allows the circuit-specific hyperactivity to diminish. Over time, continued stress management through social support, a structured routine, regular exercise, and normal sleep can reduce and, in some cases, even eliminate the need for medication, which again indicates that psychotropic drugs and stress reduction are working through the same mechanism. Then again, the higher the baseline level of neuronal excitability, the more likely that long-term medication maintenance will be needed. All of this is in keeping with the idea that it is not the brain that suffers emotional breakdowns but the person; people break down when the number of inputs from pathologically hyperactive neural circuits becomes too overwhelming to handle mentally.

Another illness category that can readily be explained by the MCNH hypothesis is that of substance use disorders. Although a large number of persons who experiment with drugs and alcohol eventually become addicted, most do not. While there are numerous factors that help shape the trajectory of a person's drug use, two consistent observations combine to suggest that an individual's baseline level of neuronal excitability is the most important of them. The first observation is that alcohol and marijuana, the two most commonly abused drugs, have potent anticonvulsant effects. Stimulant-type drugs are actually less preferred despite the fact that they activate the reward system of the brain. In a recent survey conducted by the Substance Abuse and Mental Health Services Administration [75], only $25 \%$ of those who had used crack cocaine in the previous one-to-two-year period had reused one year later. In contrast, more than half of those who had used marijuana, and roughly three quarters of those who had used alcohol, had reused during the same follow-up period. What makes sedatives so attractive is that they reduce the neuronal excitability that theoretically drives most illicit drug use. Even when addicts do use stimulants, they generally prefer to combine them with sedatives in an effort to prevent the stimulants from exacerbating the anxiety, irritability, and other symptoms that are driven by hyperactivity in the associated circuit loops. A well-known class of drugs that helps reduce circuit-specific hyperactivity while at the same time activating the brain's reward system is the opium alkaloids. This partially explains why opioids are so powerfully addictive. The second observation that links neuronal hyperexcitability to substance abuse is the high comorbidity between psychiatric disorders and substance use disorders. According to reports published in the Journal of the American Medical Association [76], 37\% of those who abuse alcohol, and $53 \%$ of those who abuse other drugs have at least one serious mental health condition. The emphasis here is on the word "serious." Assuming that those with serious mental health conditions are just the tip of the iceberg, then many more must have mild to moderate mental health conditions. That tells us that virtually all persons who abuse alcohol or other drugs have some type of mental health condition. If we now return to the hypothesis that neuronal hyperexcitability is at the root of psychiatric disorders, it becomes apparent that an inherent hyperexcitability of the neurological system is the biggest risk factor for substance abuse.

Drugs and alcohol are not the only addictions that are proposed to be rooted in neuronal hyperexcitability. Virtually all addictive behavior, whether it be gambling, sex, pornography, or some other obsessive behavior is in theory driven by the reverberating circuit loops that are fueled by the common but elusive trait of neuronal hyperexcitability. The reward-seeking behaviors that these individuals repeatedly engage in are, from a psychophysiological perspective, desperate attempts to counterbalance excessive activity in dysphoria-producing circuitry by bolstering 
activity in euphoria-producing circuitry. As predicted by the hypothesis, these non-drug addictions, like chemical addictions, are frequently associated with other psychiatric symptoms and diagnoses.

\subsection{Neuropsychiatric Evidence}

Yet another observation that links psychiatric disorders to neuronal hyperexcitability is the exacerbation of psychiatric symptoms by inflammation. Inflammatory cytokines increase neuronal excitability by several mechanisms [77-79], thus helping to explain the psychiatric manifestations of autoimmune diseases, infectious diseases, allergens, and any process that triggers an immune response [80]. The growing recognition that inflammation can precipitate or exacerbate psychiatric symptoms has led some to speculate that mental illness is an inflammatory disease. However, only a subset of patients develop psychiatric symptoms in association with inflammation, and reviews of studies in which antiinflammatory drugs were used to treat major psychiatric disorders such as schizophrenia and major depression found that these agents, while sometimes being helpful, generally failed to eliminate all of the symptoms [16, 17, 81]. According to the neuronal hyperexcitability hypothesis, that is because they reduce the inflammation that is merely exacerbating the underlying neuronal hyperexcitability. As predicted by the hypothesis, anti-inflammatory drugs have been of most benefit in those patients who have the most pretreatment inflammation $[16,82]$.

\subsection{Behavioral Evidence}

Neuronal hyperexcitability is also proposed to be at the root of personality disorders. What distinguishes a "personality disorder" from normal personality traits are the primitive defense mechanisms, rigidity of thinking, and selfdefeating behaviors that personality-disordered individuals develop from an early age and tend to maintain throughout the course of their lives. Because neuronal hyperexcitability amplifies every life experience, it increases the perceived magnitude of every stressful event. In an effort to protect the self, affected individuals are apt to construct elaborate defense mechanisms and become behaviorally inappropriate, particularly if their baseline level of neuronal excitability is exceptionally high and they are raised in a stressful environment. More often than not, these two risk factors cooccur because, as will become apparent, the trait of neuronal hyperexcitability runs in families, and in its severe form, tends to promote emotional volatility, substance abuse, and domestic violence.

Then again, the vulnerability trait appears to weigh far more heavily than environmental factors in the development of psychiatric symptoms. A careful analysis of the pedigrees of personality-disordered individuals clearly indicates that many of these families include individuals who, despite being raised by the same parents in the same households, are completely free of the emotional and behavioral problems that plague their siblings. This observation, taken together with the early childhood development of personality disorder traits, tells us two things: 1) that the trait of neuronal hyperexcitability is present from an early age; and 2) that it is the most important of the variables that bear on the development of a personality disorder.

These observations would also explain why the comorbidity between personality disorders and other psychiatric disorders (formerly referred to as Axis I disorders) is so high and why the related Axis I disorder often contains elements of the associated personality disorder. For example, persons with schizotypal personality disorder have so many characteristics of schizophrenia that the two disorders can be virtually indistinguishable. In borderline personality disorder, a condition that is characterized by emotional instability, the rate of a cooccurring mood or anxiety disorder is around 90\% [83]. From the perspective of the $\mathrm{MCNH}$ hypothesis, the close relationship between personality disorders and their related Axis I disorders is not coincidental; it's as if the brain were training the mind to habitually mimic and permanently adopt the thoughts, feelings, and behaviors that are driven by the related circuit-specific imbalances. The relatively high severity and chronicity of the circuit-specific imbalances in personality-disordered individuals would also explain why antidepressants, which can further excite parts of the brain [84-88], tend to be much less effective for personalitydisordered individuals than anticonvulsant and antipsychotic drugs [89-91]. This, taken together with the high level of comorbidity and large overlap in symptomatology between personality disorders and Axis I disorders, strongly suggests that the two disorder-types are rooted in the same biological vulnerability trait; namely, neuronal hyperexcitability.

\subsection{Medical Evidence}

In the same way that neuronal hyperexcitability can drive the development of Axis I and Axis II disorders, it can drive the development of the functional somatic disorders that are commonly associated with psychiatric disorders, such as recurrent headaches, fibromyalgia, and irritable bowel syndrome (IBS). Like psychiatric symptoms, the physical symptoms that characterize these disorders tend to wax and wane in severity and become exacerbated by stress. At times in the course of the illness they may be accompanied by psychiatric symptoms, and at other times they may alternate with psychiatric symptoms. What theoretically happens neurologically is that the locus of hyperactivity wanders from one set of circuits to another, sometimes activating cognitive circuits, sometimes activating emotional circuits, and sometimes activating circuits that provide the signaling to and from the body parts that are identified as being symptomatic. Exactly where the locus of hyperactivity roams and for how long it persists depends on a dynamic interplay between the mind, the brain, and the natural tendency for various circuit loops to compete for dominance when the neurological system is pathologically hyperactive [68].

In recent years, there has been a growing awareness that certain foods may contribute to the symptoms of these 
functional disorders. The theoretical reason that dietary changes such as carbohydrate restriction (particularly the avoidance of refined sugar) and reduction of other potential mediators of inflammation, such as gluten, casein, and lectins, can reduce both functional somatic and neuropsychiatric symptoms is that they reduce the level of neuronal excitability [19, 92-98]. As the brain calms down, there is less of the electrical activity that theoretically drives the development of migraine headaches [99, 100], and less of the top-down stimulation of skeletal and smooth muscle that theoretically drives the symptoms of tension headaches, fibromyalgia, IBS, and related disorders [101]. Additional support for the idea that diet can reduce symptoms by altering brain physiology comes from the cognitivebehavioral benefits that have been observed in autism spectrum disorders through attention to the gut-brain axis [102].

Because neuronal hyperexcitability also amplifies the stress response, circulating levels of epinephrine and cortisol can be chronically elevated (Figure 7). In addition to increasing heart rate, blood pressure, and respiratory rate, epinephrine combines with cortisol to stimulate the release of glucose, fatty acids, and cholesterol into the blood. It is the endogenous release of these nutrients, more than dietary intake, and the accompanying stress-induced inflammation, more than the nutrients themselves, that leads to both the development of atherosclerotic plaques and the rupture of those plaques [103]. Like many diseases that are believed to be inflammatory in nature, atheroschlerosis is increasingly coming to be known as an inflammatory disease.

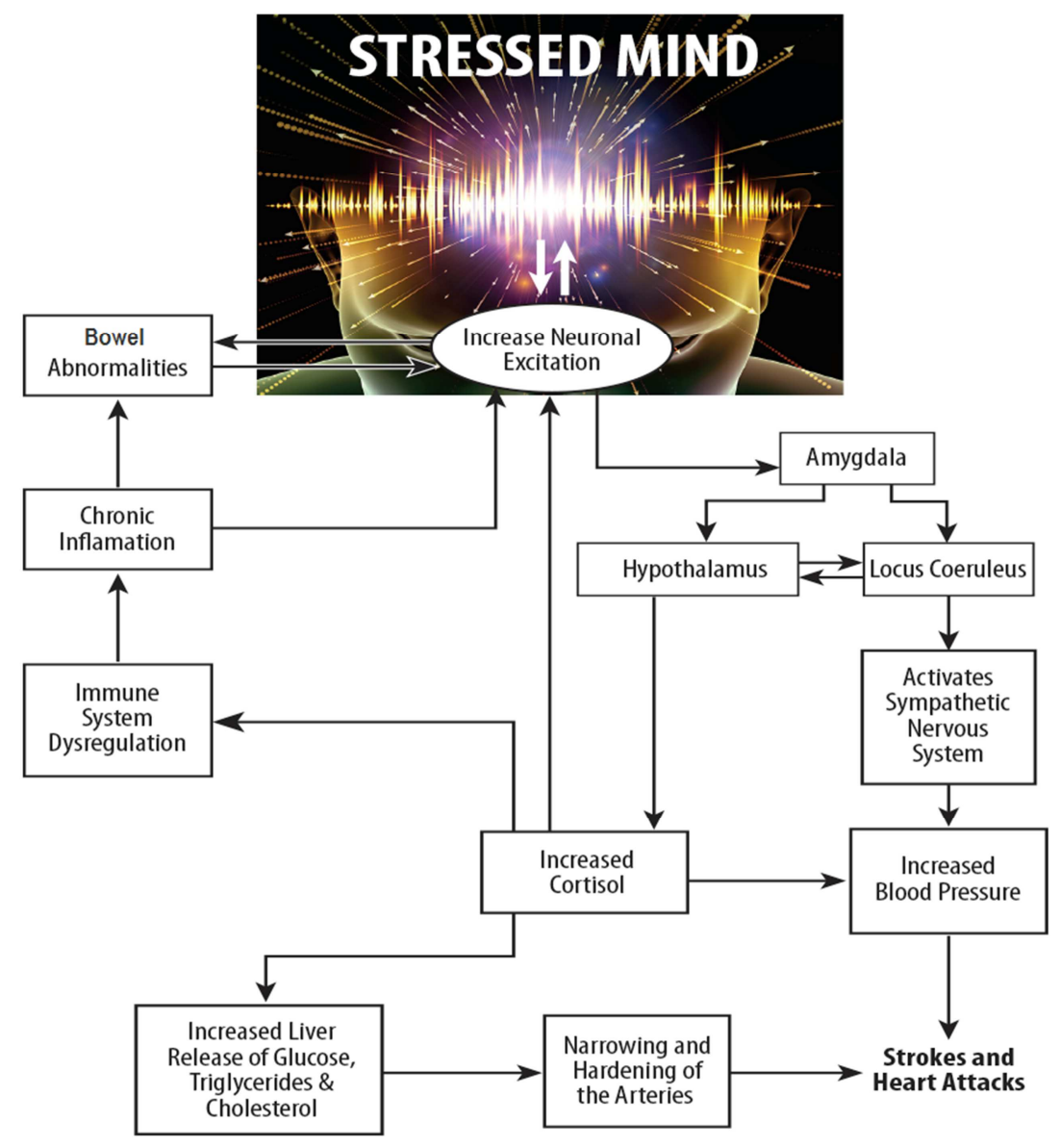

Figure 7. Flow diagram illustrating the psychophysiology of chronic stress.

The most ubiquitous inducer of inflammation is chronic stress [104, 105]. Hence, persons with neuronal hyperexcitability, who by definition have an inherent tendency to overreact to stress, would be at increased risk of suffering the physical effects of chronic inflammation. This, together with the unhealthy lifestyles and dietary habits that are driven by chronic stress, could explain why certain emotional response patterns and personality types have been associated with an elevated risk of cardiovascular disease. The prototypical example of this is the so-called "type-A personality," which has long been associated with an increased risk of hypertension and coronary artery disease [106]. The experience of chronic stress also tends to cause chronic muscle tension. This not only contributes to the functional pain syndromes that are associated with psychiatric disorders but also hastens the development of hypertonic spasm, which is an imperceptible but permanent contraction of skeletal muscle that, in its advanced stages, is the fundamental physical component of chronic pain [107].

Clearly, the profound effects that chronic stress can have on the general health of the body make the identification of neuronal hyperexcitability as important medically as it is 
psychiatrically. Moreover, the observation that most visits to the primary care doctor are driven by stress-related functional disorders illustrates how common the trait of neuronal hyperexcitability is in comparison to other organic abnormalities.

\subsection{Psychophysiological Evidence}

While the neuronal hyperexcitability hypothesis can readily explain most of what is observed clinically in psychiatric disorders, there is one seeming exception. Persons with unipolar depression do not, from a clinical standpoint, appear to have hyperactive brains; on the contrary, their brains appear to be slowed down. What one must remember, however, is that the depressed mind has little interest in doing anything. Consistent with this conceptualization, the MCNH hypothesis predicts that hyperactivity in depressive circuit loops would rob clinically depressed patients of all that is of interest in life and trap them in a world of misery, thus explaining why some of them just lie in bed all day and ruminate about dying.

When such patients are assessed, the idea that their brains could be hyperactive is the furthest thing from the clinician's mind. What these patients seem to need is something to pep them up; hence the logic of prescribing drugs that have stimulatory effects. However, contrary to what these patients may look like clinically, their symptoms, like those of other psychiatric disorders, are in theory driven by neuronal hyperactivity [108]. In the case of clinical depression, the hyperactivity simply involves depressive circuit loops [109].

Until recently, constraints in neuroimaging had yielded results that seemed to support the clinical impression that depressed patients were slowed down neurologically. When regional metabolic activity was studied in depressed patients, the circuit-specific hyper-metabolism that would have been predicted by the MCNH hypothesis was overshadowed by a more generalized hypo-metabolism consequent to the patient's general state of cognitive-emotional withdrawal $[110,111]$. What theoretically happens in clinical depression is that the mind gets caught in negative ruminations, and the attention paid to the dysphoric content causes circuits for goal-directed thinking to be subdued $[112,113]$. Were it not for this modulatory mechanism, one would not be able to concentrate on one thing at a time. Moreover, the more the mind gets caught in a specific cognitive-emotional state, the more that competing circuit loops tend to be inhibited [112].

Then again, many patients who suffer from clinical depression are not physically slowed down; on the contrary, they are physically sped up. They are restless, irritable, and unable to sleep. What theoretically distinguishes one form of depression from another is the specific distribution and degree of persistence of the related circuit-specific imbalances. Also of some influence is the way that the individual responds to this activity mentally. One of the things that make psychiatric disorders so distressing is that the overabundance of neurological activity bombards the mind with unintended thoughts and feelings like spam on a computer. For many persons with neuronal hyperexcitability, this can overwhelm the modulatory function of the thalamic reticular nucleus (TRN) $[112,114-116]$, prompting the individual to respond to various thoughts and feelings before the brain can fully process them. This may be expressed clinically as inattention, hyperactivity, and impulsivity, thus explaining why attention deficit hyperactivity disorder (ADHD) is such a common comorbidity in psychiatric disorders. What allows psychostimulants to help alleviate ADHD symptoms is that both dopamine and norepinephrine increase the output of TRN collaterals [114, 115], which, being primarily inhibitory, act as circuit-breakers that help reduce the volume of extraneous electrical activity in the brain. With less information popping up on the thalamic computer screen, the mind is better able to focus on one thing at a time.

A consistent clinical finding in patients with psychiatric disorders is that they experience an overabundance of thoughts and feelings even when they are relatively asymptomatic. While a normal person may experience just one or two random thoughts and feelings over a brief period of time, a person with a psychiatric history may experience ten or more random thoughts and feelings over the same period of time. Moreover, this can change as the locus of hyperactivity shuttles between cognitive and limbic circuitry. That the associated thoughts and feelings emanate from a wide variety of circuits suggests that the trait of neuronal hyperexcitability is a generalized physiological abnormality, though the specificity of the firing imbalances and the tendency for the associated symptoms to morph into one another can create the false impression that they represent a diversity of biological abnormalities.

\subsection{Experimental Evidence}

Experimental evidence that clinical depression involves hyperactivity in depressive circuit loops comes from a study by Mazarati et al, who found that when the level of neuronal excitability was experimentally increased by repeated subconvulsive stimulation of the brain, the laboratory animals began to demonstrate depressive-like behavior [23]. This observation, taken together with the observation that depressive symptoms in susceptible individuals commonly develop in association with the circuit-specific stimulatory effects of severe or recurrent psychosocial stress [117], is compelling evidence that clinical depression is a manifestation of hyperactivity in depressive brain circuitry. Lending further support to this hypothesis is the observation that the increased vulnerability to depression that is fueled by persistent psychosocial stress persists for about the same length of time as an experimentally-induced kindling effect [118].

\subsection{Radiological Evidence}

That clinical depression and other psychiatric disorders are associated with neuronal hyperactivity is becoming increasingly apparent with the development of more sophisticated neuroimaging techniques. In one of the first 
studies of its kind, Johnstone et al, using fMRI technology, found that the brains of persons who suffer from clinical depression had an inability to regulate negative mood when it arose [108]. Unlike normal persons, who were able to consciously regulate activity in their emotional response centers, depressed subjects continued to display high activity in their emotional centers despite intense activity in the regulatory regions of the brain. Moreover, the processing abnormalities became more pronounced the harder the depressed subjects tried to regulate their emotions. As expected, their mental effort had a stimulatory effect on the brain, which itself was feeding into the underlying problem of neuronal hyperexcitability. Moreover, because the effort inevitably stimulated circuits that were already symptomatically hyperactive, it rendered their efforts counter-productive.

More recently, Leuchter et al found that clinically depressed subjects showed increased synchronization across all frequencies of electrical activity, indicating dysfunction in many different brain networks [109]. According to Dr. Leuchter, the healthy brain must be able to first synchronize and then desynchronize activity from various brain areas in order to allow us to analyze information, regulate mood, and control our actions. In persons with clinical depression, multiple regions of the brain showed hyperactivity, hyperconnectivity, and hypersynchronization, indicating that electrical signals would not shut off. The area of the brain that showed the most severe abnormalities was the prefrontal cortex, which works in conjunction with the limbic system to regulate mood and solve problems. This observation is consistent with what was found in the Johnstone study and reiterates what was said earlier, which was that the surges of emotion that are induced by neuronal hyperexcitability create a vicious cycle of overstimulation in the circuit loops that generate the related psychiatric symptoms.

In comparable studies involving pain, researchers have found that chronic pain patients react to noxious stimuli more strongly and for longer periods of time than normal subjects [119]. Functional imaging studies show distinctly elevated activity in the brain areas related to pain perception [120], and virtually all such studies have shown that chronic pain and its comorbid psychiatric symptoms are associated with alterations in neurological activity across several brain regions, with no one region of the brain, brainstem, or spinal cord singularly responsible for the persistent pain [121]. Consistent with the neuronal hyperexcitability hypothesis, this provides evidence that in some persons, neurons throughout the nervous system are hyperexcitable and won't shut off.

\subsection{Explanatory Evidence}

Although most chronic and recurrent psychiatric disorders are proposed to be rooted in an inherent hyperexcitability of the neurological system, not every person with neuronal hyperexcitability will develop the same psychiatric or somatic symptoms. In fact, some persons might not develop any symptoms at all. That is because there are a number of other factors that affect the nature, severity, and degree of persistence of an individual's symptoms. These include the severity of the stressors encountered, one's personal attitude, and the effectiveness of one's coping skills.

Chief among these, however, is the excitability of the neurological system because neuronal hyperexcitability tends to amplify all forms of perceived stress. The greater the excitability, the greater the perceived stress. Stress creates intrapsychic tension, which in turn directly and intensely excites the circuit loops that are associated with the stressor. This further amplifies the perceived stress. Thus, stress tends to beget more stress, especially if the neurological system is hyperexcitable. In this sense, the hyperexcitable brain can be compared to a hive of irritable bees. When stressed, the bees easily become agitated and begin to swarm around the hive, causing the one who is agitating them to try to shoo them away. This further agitates the bees, thus creating a vicious cycle of mutual provocation between the bees and the one who is agitating them. So it is with the hyperexcitable brain in relation to the stressed mind.

The first major wave of stress in a person's life typically occurs during the transition from childhood to adulthood, thus explaining why adolescence is the time that psychiatric symptoms are most likely to emerge. Then again, adolescence is not always the first high-stress period in a person's life. For some it might be early childhood; for others it might be the college years; and for still others it might be retirement or beyond. Moreover, potential precipitating factors are not limited to negative stressors; positive stressors can have the same effect, as can biological stressors such as hormonal fluctuations, inflammatory processes, stimulanttype drugs, withdrawal from sedatives, or any biological factor that has a stimulating effect on the brain. Also of some influence but of lesser significance are the structural brain changes that occur as a person ages [122].

Thus, the neuronal hyperexcitability hypothesis provides a precise, psychophysiological explanation for why psychiatric symptoms are frequently but not always age-related. It also explains why psychosocial stressors are the most ubiquitous precipitant of psychiatric symptoms. Chief among these is relationship stress, which among other personal stressors, some patients may be hesitant to disclose. This can create the impression that some psychiatric episodes occur spontaneously. However, excluding those cases in which there is a complicating biological stressor, a careful analysis of the illness history of psychiatric patients consistently reveals a clear, albeit delayed, temporal relationship between the onset of an objective psychosocial stressor and the development of symptoms. Typically, the onset of symptoms will be delayed by days, weeks, or months, which is the time required for the stressed mind to induce enough kindling in the brain to precipitate symptoms. What makes some individuals more vulnerable to this effect is that their neurons are inherently hyperexcitable. This is critically important to recognize, for although psychological interventions, such as stress reduction, meditation, and psychotherapy, can have a calming effect on the brain, they do not change the 
temperament of the bees; they merely help prevent them from becoming perturbed. Similarly, dietary changes such as the avoidance of caffeine, elimination of refined sugar, and attention to potential mediators of inflammation, while helping to prevent the brain from becoming chemically stimulated, do not change the inherent excitability of the neurons.

\section{Etiology of the Vulnerability Trait}

Although extensive data from family, twin, and adoption studies indicate that psychiatric disorders and their common comorbidities have a significant genetic component, individual disorders fail to follow a consistent pattern of inheritance. However, if one considers the varying degrees to which the trait for neuronal hyperexcitability can be expressed and the diversity of forms that its expression can take, one could not reasonably expect the same symptomatology to be passed from one generation to the next even if the same gene variants were inherited. If, with this in mind, we go back and reconstruct family pedigrees based not only on overt psychiatric symptoms but also on soft signs of neuronal hyperexcitability, such as hyper-emotionality, mood instability, sleep difficulties, attentional problems, functional somatic complaints, and substance use disorders, a consistent pattern of distribution emerges; that pattern is strikingly autosomal dominant (Figure 8).

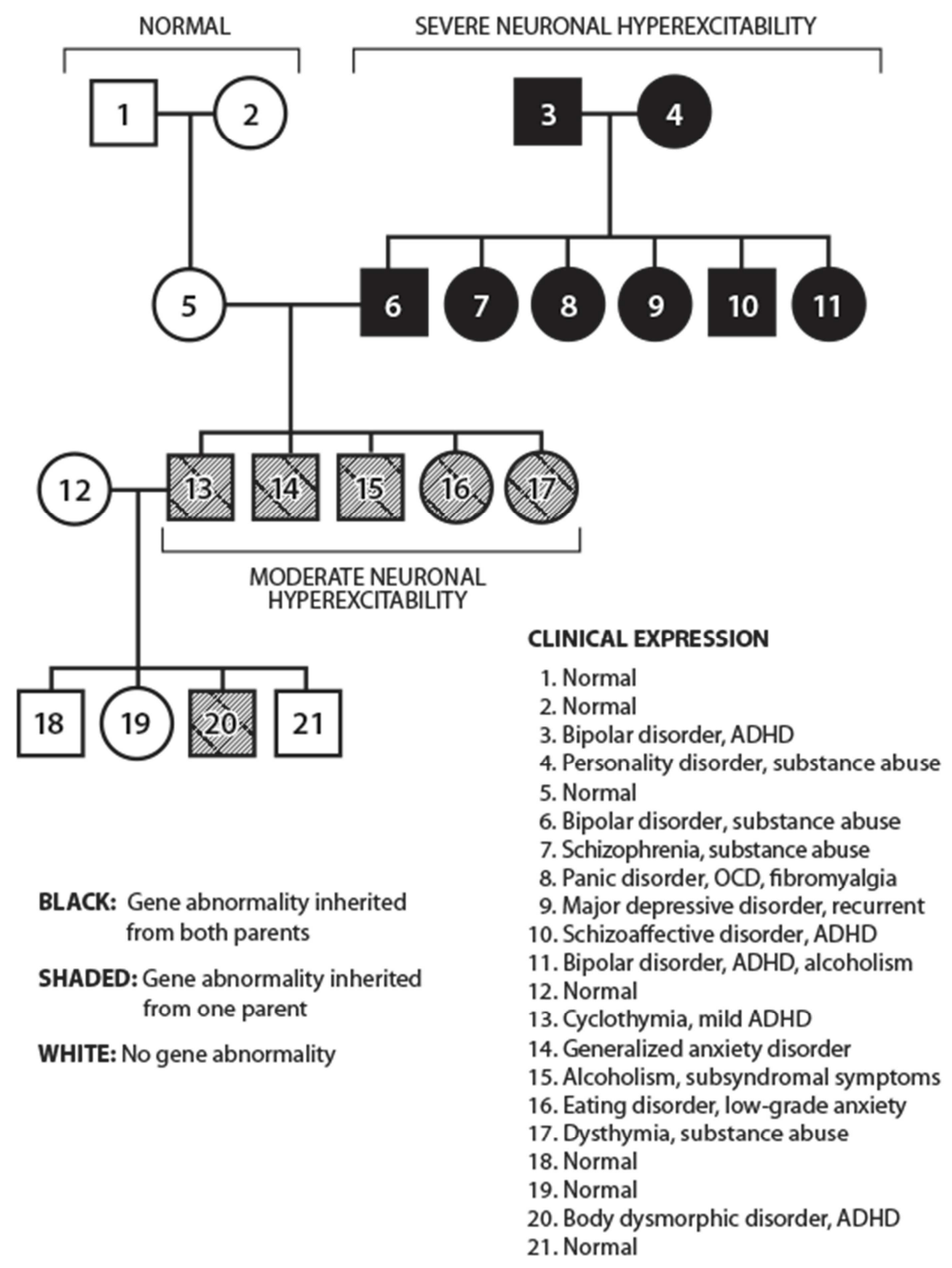

Figure 8. Pedigree chart illustrating the inheritance pattern of the neuronal hyperexcitability trait and the various phenotypic expressions of the trait. Note that some of those who inherit one allele might simply self-medicate or have symptoms that are too subtle to meet criteria for one of the currently recognized psychiatric disorders. Those who inherit two alleles typically have more obvious psychiatric symptoms. Also note that the inheritance of the susceptibility genes can manifest as a functional somatic disorder. Distribution pattern is based on more than one hundred consecutive clinical interviews. Formal diagnostic criteria were intentionally not applied. 
The validity of this finding is further supported by the observation that a predictable proportion of individuals in these families will be completely free of psychiatric symptoms irrespective of how dysfunctional their family dynamics might be. These so-called "survivors," who appear in a classic autosomal recessive distribution, are not necessarily more mentally tough than their siblings, but rather more neurologically stable presumably because they did not inherit one of the gene variants that have been linked to neuronal hyperexcitability. Moreover, the fact that these individuals are so resistant to illness tells us that among the variables that contribute to the development of psychiatric disorders, the trait of neuronal hyperexcitability is by far the most important. Additionally, the autosomal dominant pattern of inheritance and sharp clinical distinction between those who inherent the susceptibility genes and those who do not suggest that most of the candidate genes that have been linked to psychiatric disorders make small contributions in comparison to a few genes that make large contributions and may by themselves be enough to markedly increase one's vulnerability to mental illness.

\section{The Gene Research}

Based on the classic Mendelian distribution of the various manifestations of the neuronal hyperexcitability trait, one could anticipate three things: 1) that psychiatric disorders would be highly correlated with specific gene polymorphisms; 2) that the most influential gene variants would be located on autosomes; and 3) that the protein products of those gene variants would be involved in the regulation of neuronal excitability. In turning to the gene research, that is exactly what was found.

In the largest study of its kind, a collaborative research group involving institutions from across the United States performed a genome-wide association analysis in search of susceptibility loci for bipolar disorder [123]. Bipolar disorder can be thought of as a kind of clinical umbrella that contains elements of most of the major psychiatric disorders. Symptoms of bipolar disorder may include periods of depression that characterize major depressive disorder; periods of euphoria that characterize mania; rapid cycling that characterizes cyclothymia; mood instability that characterizes borderline states; generalized anxiety that characterizes anxiety disorders; panic attacks that characterize panic disorder; psychotic states that characterize schizophrenia; anger outbursts that characterize intermittent explosive disorder; primitive defense mechanisms that characterize personality disorders; and the triad of inattention, hyperactivity, and impulsivity that characterizes ADHD. Bipolar patients commonly also suffer from one or more functional somatic disorders, such as recurrent headaches, IBS, fibromyalgia, chronic musculoskeletal pain, or some other disorder that has been linked to a hypersensitivity of the central nervous system [99-101, 124].

The collaborative research group tested 1.8 million gene variants in 4,397 cases of bipolar disorder and 6,209 controls, and found two regions of strong association [123]. The most significant was Ankyrin 3 (ANK3) on chromosome 10q21, which codes for a protein that is found at the axon initial segment and nodes of Ranvier of neurons in the central and peripheral nervous systems [125]. The protein, which has also been linked to schizophrenia [126], has been shown to regulate the assembly of voltage-gated sodium channels [123], thereby affecting the neuron's excitability. ANK3 was also linked to epilepsy [127], a disorder that is known to be fueled by neuronal hyperexcitability.

The second strongest region of association was located in the third intron of CACNA1C on chromosome 12p13. Like ANK3, CACNA1C, which has also been linked to schizophrenia and major depressive disorder [128, 129], codes for a protein that regulates the movement of ions, in this case calcium ions, across neuronal membranes [123, 130]. Strikingly, CACNA1C is the site of another medication that is used to treat mood disorders. That medication reduces the movement of the same ions across neuronal membranes, thereby helping to compensate for the defective gene product. The same researchers found that in the mouse brain, both ANK3 and subunits of the calcium channel were downregulated in response to lithium, a drug that is known to have anticonvulsant effects [131-133], and one of the oldest medications used in the treatment of bipolar disorder, schizophrenia, and schizoaffective disorder. These unlikely connections provide a strong molecular basis of support for the neuronal hyperexcitability hypothesis of psychiatric disorders.

Among the other candidate genes that have been linked to the major psychiatric disorders, nearly all have been found to code for proteins that are involved in the regulation of neuronal excitability [134-140]. This, taken together with the aforementioned biological, observational, pharmacological, neuropsychiatric, behavioral, medical, psychophysiological, experimental, radiological, and explanatory evidence of the same linkage, is overwhelming evidence that neuronal hyperexcitability is at the root of mental illness.

\section{What About Antidepressants and the Monoamine Hypothesis of Depression}

Based on the hypothesis that psychiatric symptoms are caused by pathological elevations in circuit-specific firing, any treatment approach to clinical depression must target this imbalance. One way that an antidepressant could do this would be to increase neurotransmission in positive (feel good) neural circuitry more than negative (feel bad) neural circuitry. Alternatively, it could turn down firing in negative neural circuitry, such as when a serotonergic antidepressant reduces firing in the amygdala. Although this kind of rebalancing can be effective in some patients, the problem with antidepressants, including serotonergic antidepressants, is that they simultaneously increase excitatory transmission 
in various other parts of the brain, particularly the neocortex $[84,85]$. These excitatory effects are especially prominent with monoamine oxidase inhibitors and tricyclic antidepressants [86-88]. Consequently they, even more than serotonin reuptake inhibitors, have the potential to fan the flames of neuronal hyperexcitability. This kindling effect is what theoretically underlies the paradoxical effects, manicdepressive switching, and loss of effectiveness over time that are commonly associated with antidepressant therapy.

Yet that still begs the question: how is it possible that the concentration of monoamine metabolites can be decreased in the cerebral spinal fluid of at least some patients with clinical depression [141]? Would not one expect monoamine levels to be either normal or elevated? Again, the answer lies in the dynamics of brain circuitry. What theoretically happens in clinical depression is that persistent hyperactivity in negative (feel bad) neural circuits both directly and indirectly drives a persistent hyperactivation of a small epithalamic structure called the habenula [142-145]. Increased firing in the habenula inhibits the firing of large populations of neurons that release the "feel good" neurotransmitters serotonin and dopamine [146-148]. This makes habenular hyperactivation one possible mechanism by which the concentration of monoamines could fall in depressive states. A second mechanism would be the natural inhibition of reward circuitry as dysphoria-related circuitry is activated, thus explaining why homovanillic acid, the major breakdown product of dopamine, is the monoamine metabolite that is most consistently decreased in the cerebral spinal fluid of depressed patients [141, 149]. Although antidepressants can potentially bolster activity in the monoaminergic system, the circuit specificity that is required does not align well with the method of delivery. That the drug necessarily goes everywhere in the brain leaves a lot of room for uncertainty about how it will affect the various firing imbalances that are theoretically driving the symptoms.

A much safer and more predictable way to correct a circuit-specific imbalance, and one that aligns well with the method of delivery, is to globally reduce the electrical activity in the brain. This has traditionally been done using anticonvulsant drugs. Though anticonvulsants do not target specific circuits, their relative impact tends to be greatest on those circuits that are the most severely hyperactive and, thus, the most actively symptomatic. This leads to a generalized reduction of symptoms while at the same time reducing the excitability that makes one vulnerable to developing pathological circuit-specific imbalances in the first place. This is the MCNH explanation for why anticonvulsants are associated with a much lower risk of manic-depressive switching than antidepressants. What's more, anticonvulsants exert their therapeutic effects almost immediately because they target the root of the problem, which is the inability of neurons to self-regulate.

The therapeutic success of anticonvulsants in a wide range of psychiatric disorders bears witness to the validity of the MCNH hypothesis. Both the benzodiazepine and nonbenzodiazepines anticonvulsants have demonstrated effectiveness in the treatment of anxiety disorders, sleep disorders, and substance use disorders [150-159]. In addition, the non-benzodiazepine anticonvulsants are well-known to be effective in the treatment of bipolar disorder, which, as previously stated, is a kind of diagnostic umbrella that includes the symptoms of most of the other psychiatric disorders. What's more, numerous studies have found that patients with bipolar disorder are in most cases misdiagnosed with unipolar depression [160-162]. This observation, taken together with the fact that the more subtle disorders in the bipolar spectrum, such as bipolar II, cyclothymia, and cyclic depression, would be even more likely to be misdiagnosed with a unipolar depressive disorder, statistically points to the non-benzodiazepine anticonvulsants as the treatment of choice in the vast majority of patients who are diagnosed with major depression or dysthymia.

Beyond that, both the benzodiazepine and nonbenzodiazepine anticonvulsants have a long history of adjunctive use in the treatment of schizophrenia [163-165], and the non-benzodiazepine anticonvulsants are increasingly being used in the treatment of migraine headaches [166], fibromyalgia [167], chronic pain [168], and a host of other disorders that have been linked to a hypersensitivity of the central nervous system [99]. Lastly, both benzodiazepine and non-benzodiazepine anticonvulsants can assist in the treatment of ADHD [169, 170], and they can be an indispensable adjunct in the treatment of personality disorders, particularly when mood and anxiety symptoms are prominent [89-91].

Although anticonvulsant drugs are effective for most psychiatric disorders, their broad spectrum utility and rapid onset of action have been overshadowed by the stimulating effects of antidepressants. When the "energizer effect" was serendipitously discovered in the 1950s, the patients in the tuberculosis sanatorium who might have been depressed were experiencing more than a normalizing effect from their antituberculin drugs; they were experiencing psychostimulation and a mild euphoria that, in some cases, included hypersexuality and psychotic symptoms [171, 172]. An Associated Press release from Staten Island's Seaview Hospital captured a telling scene: patients dancing in celebratory mood [173]. That is more than a mood-normalizing effect.

Hence, if an anticonvulsant, which normalizes brain function in those who are vulnerable to mental illness, were to be compared head-to-head with an antidepressant, which preferentially boosts activity in "feel-good" neural circuits, the antidepressant would tend to outperform the anticonvulsant. The problem is that the vast majority of clinically depressed patients have significant neuronal hyperexcitability, which is what makes them vulnerable to depression in the first place. For such patients, the stimulating effects of an antidepressant can further destabilize the electrical system of the brain, which is why antidepressants have such a high propensity for paradoxical effects. The trait of neuronal hyperexcitability would also explain why unmedicated remitted patients, unlike controls, experience a return of depressive symptoms in response to 
experimentally-induced serotonin depletion [174]. As would be predicted by the MCNH hypothesis, the inherent hyperexcitability of their neurological systems renders them intolerant of the withdrawal of serotonin's neuromodulatory effects, which at normal physiological levels, are relatively free of the stimulatory effects that may occur as an antidepressant bolsters serotonergic activity. There is also evidence that the degree of relapse in serotonin-depleted patients can be a prognostic indicator of their future response to antidepressant therapy [175]. This too is to be expected, as those with the highest sensitivity to relapse would theoretically have the highest levels of neuronal excitability and, thus, the highest propensity for paradoxical effects when treated with an antidepressant. These observations, taken together with the growing recognition that depression and other psychiatric symptoms can rapidly be reversed by novel pharmacological agents and somatic treatments that modulate excitatory neurotransmission [31, 33-36, 38, 55, 57, 142], points back to the idea that the biochemical changes that have been linked to psychiatric disorders are not the cause of the symptoms but the consequence of the circuit-specific firing imbalances that are fueled by the highly prevalent but elusive trait of neuronal hyperexcitability.

\section{Conclusion}

In summary, there is a wide body of multidisciplinary evidence that psychiatric disorders and their functional comorbidities are rooted in a single, shared, neurophysiological abnormality and that what distinguishes one disorder from another is the severity and distribution of pathological circuit-specific imbalances in the brain. Based on recent genetic findings, that abnormality appears to be rooted in nucleotide polymorphisms that code for proteins that fail to regulate the excitability of neurons.

From the study of epilepsy, it is known that brain health requires a healthy balance between excitatory and inhibitory neural signaling. Based on the MCNH hypothesis, the fastest, safest, and most direct way to treat most psychiatric disorders would be to reduce the neuronal excitability that disrupts this balance and drives the production of psychiatric symptoms. The recognition of this demystifies mental illness and provides a strong basis for studying the use of anticonvulsants and other neuromodulators in a broader range of psychiatric disorders.

To my knowledge, the MCNH hypothesis is the first to provide a molecularly-based, psychophysiologically-specific formulation for a group of disorders that continues to elude medical understanding. What has been identified as the vulnerability trait in mental illness stands to simplify diagnostics, streamline treatment, and guide a higher quality of care for what has been called "the world's largest single health problem."

\section{Competing Interests}

The author declares that the research was conducted in the absence of any commercial or financial relationships that could be construed as a potential conflict of interest.

\section{References}

[1] Wang S, Tudusciuc O, Mamelak AN, et al. (2014) Neurons in the human amygdala selective for perceived emotion. PNAS. 111 (30): E3110-E3119.

[2] Wang S, Yu R, Tyszka JM, et al. (2017) The human amygdala parametrically encodes the intensity of specific facial emotions and their categorical ambiguity. Nature Communications. 8: 14821.

[3] Jimenez JC, Su K, Goldberg AR, et al. Anxiety Cells in a hippocampal-hypothalamic circuit. Neuron. 2018; 97 (3): 670683. e6.

[4] Yizhar O, Fenno LE, and Deisseroth K. (2011) Neocortical excitation/inhibition balance in information processing and social dysfunction. Nature. 477: 171-178.

[5] Beyeler A, Namburi P, Glober GF, et al. (2016) Divergent routing of positive and negative information from the amygdala during memory retrieval. Neuron. 90: 348-361.

[6] Penfield W. (1936). Epilepsy and surgical therapy. Archives of Neurology and Psychiatry. 36 (3): 449-484.

[7] Fuchs E and Flügge G. (2003) Chronic social stress: effects on limbic brain structures. Physiology \& Behavior. 79 (3): 417427.

[8] Mehler B, Reimer B, Coughlin JF, and Dusek JA. (2009) Impact of Incremental Increases in Cognitive Workload on Physiological Arousal and Performance in Young Adult Drivers. Transportation Research Record: Journal of the Transportation Research Board. (2138): 6-12.

[9] Rose GM, Diamond DM, Pang K, and Dunwiddie TV. (1988) Primed Burst Potentiation: lasting synaptic plasticity invoked by physiologically patterned stimulation. In: Haas HL and Buzsàki G. (eds) Synaptic Plasticity in the Hippocampus. Springer, Berlin, Heidelberg.

[10] Bromfield EB, Cavazos JE, Sirven JI, editors. (2006) In: An introduction to epilepsy. West Hartford (CT): American Epilepsy Society. Chapter 1: Basic Mechanisms Underlying Seizures and Epilepsy.

[11] McKee HR and Privitera MD. (2017) Stress as a seizure precipitant: Identification, associated factors, and treatment options. Seizure. 44: 21-26.

[12] Lawn N, Lieblich S, Lee J, and Dunne J. (2014) Are seizures in the setting of sleep deprivation provoked? Epilepsy \& Behavior. 33: 122-125.

[13] Coffey CE, Figiel GS, Weiner RD, and Saunders WB. (1990) Caffeine augmentation of ECT. American Journal of Psychiatry. 147 (5): 579-585.

[14] Winston AP, Hardwick E, and Jaberi N. (2005) Neuropsychiatric effects of caffeine. Advances in Psychiatric Treatment. 11 (6): 432-439.

[15] Vezzani A. (2005) Inflammation and epilepsy. Epilepsy Curr. 5 (1): 1-6. 
[16] Bowcut JC and Weiser M. (2018) Inflammation and schizophrenia. Psychiatric Annals. 48 (5): 237-243.

[17] Raison CL and Miller AH. (2015) Anti-Inflammatory agents as antidepressants: Truth or Dare. Psychiatric Annals. 45 (5): 255-261.

[18] Bough KJ and Rho JM. (2007) Anticonvulsant mechanisms of the Ketogenic Diet. Epilepsia. 48 (1): 43-58.

[19] Bostock ECS, Kirkby KC, and Taylor BVM. (2017) The current status of the Ketogenic Diet in psychiatry. Front Psychiatry. 8 (43): 1-40.

[20] Ingram J, Zhang C, Cressman JR, et al. (2014) Oxygen and seizure dynamics: I. experiments. J Neurophysiol. 112 (2): 205-212.

[21] Hüfner K, Brugger H, Kuster E, et al. (2018) Isolated psychosis during exposure to very high and extreme altitude characterization of a new medical entity. Psychological Medicine. 48 (11): 1872-1879.

[22] Goddard GV. (1967) Development of epileptic seizures through brain stimulation at low intensity. Nature. 214: 1020-1021.

[23] Mazarati A, Shin D, Auvin S, Caplan R, and Sankar R. (2007) Kindling epileptogenesis in immature rats leads to persistent depressive behavior. Epilepsy Behav. 10: 377-383.

[24] Finocchi C and Ferrari M. (2011) Female reproductive steroids and neuronal excitability. Neurol Science. 32 (Suppl 1): 31-35.

[25] Luoma JI, Stern CM, and Mermelstein PG. (2012) Progesterone inhibition of neuronal calcium signaling underlies aspects of progesterone-mediated neuroprotection. The Journal of Steroid Biochemistry and Molecular Biology. 131 (1-2): 30-6.

[26] Stein DG. (2008) Progesterone exerts neuroprotective effects after brain injury. Brain Research Reviews. 57 (2): 386-97.

[27] Devinsky O, Cilio MR, and Friedman D. (2014) Cannabidiol: Pharmacology and potential therapeutic role in epilepsy and other neuropsychiatric disorders. Epilepsia. 55 (6): 791-802.

[28] Sankaranarayanan A, Wilding H, Neill E, and Castle D. (2018) A critical systematic review of evidence for cannabinoids in the treatment of schizophrenia. Psychiatric Annals. 48 (5): 214-213.

[29] Zuardi A, Shirakawa I, Finkelfarb E, and Karniol I. (1982) Action of cannabidiol on the anxiety and other effects produced by delta-9-THC in normal subjects. Psychopharmacology (Berl). 76 (3): 245-250.

[30] Papanicolaou J, Summers RJ, Vajda FJ, and Louis WJ. (1982) Anticonvulsant effects of clonidine mediated through central alpha2-adrenoceptors. Eur J Pharmacol. 77 (2-3): 163-166.

[31] Kontaxakis V, Markianos M, Markidis M, and Stefanis C. (1989) Clonidine in the treatment of mixed bipolar disorder. Acta Psychiatrica Scandinavica. 79 (1): 108-110.

[32] Farook JM, Krazem A, and Barron S. (2008) Acamprosate attenuates the handling induced convulsions during alcohol withdrawal in Swiss Webster mice. Physiol Behav. 95 (1-2): 267-279.

[33] Mason BJ and Heyser CJ. (2010) Acamprosate: a prototypic neuromodulator in the treatment of alcohol dependence. CNS
Neurol Disord Drug Targets. 9 (1): 23-32.

[34] Maremmani AGI, Bacciardi, S, and Maremmani I. (2014) Sixmonth outcome in bipolar spectrum alcoholics treated with acamprosate after detoxification: a retrospective study. Int J Environ Res Public Health. 11 (12): 12983-12996.

[35] Hertzman M, Patt IS, and Spielman LA. (2009) Open-label trial of acamprosate as a treatment for anxiety. Prim Care Companion J Clin Psychiatry. 11 (5): 267.

[36] Schwartz TL, Siddiqui UA, Raza S, and Costello A. (2010) Acamprosate calcium as augmentation therapy for anxiety disorders. Annals of Pharmacotherapy. 44 (12): 1930-1932.

[37] Borowicz KK, Sêkowski A, Drelewska E, and Czuczwar SJ. (2004) Riluzole enhances the antiseizure action of conventional antiepileptic drugs against pentetrazole-induced convulsions in mice. Pol J Pharmacol. 56: 187-193.

[38] Zarate C. (2008) Riluzole in psychiatry: a systematic review of the literature. Expert Opin Drug Metab Toxicol. 4 (9): 1223-1234.

[39] Deepmala, Slattery J, Kumar N, et al. (2015) Clinical trials of $\mathrm{N}$-acetylcysteine in psychiatry and neurology: A systematic review. Neuroscience and Biobehavioral Reviews. 55: 294321.

[40] Standley CA, Irtenkauf SM, and Cotton DB. (1995). Anticonvulsant effects of magnesium sulfate in hippocampalkindled rats. Journal of Biomedical Science. 2 (1): 57-62.

[41] Eby GA and Eby KL. (2006) Rapid recovery from major depression using magnesium treatment. Medical Hypotheses. 67 (2): 362-370.

[42] El Idrissi A, Messing J, Scalia J, and Trenkner E. (2003) Prevention of epileptic seizures by taurine. Advances in Experimental Medicine and Biology. 526: 515-25.

[43] Kong WX, Chen SW, Li YL, et al. (2006) Effects of taurine on rat behaviors in three anxiety models. Pharmacol Biochem Behav. 83 (2): 271-276.

[44] Lundberg L. (2011) A modeling study of effects of polyunsaturated fatty acids, on neuronal excitability: implications in epilepsy. Master's Thesis in Computer Science at Stockholm University, Sweden.

[45] Peet $M$ and Stokes C. (2005) Omega-3 fatty acids in the treatment of psychiatric disorders. Drugs. 65 (8): 1051-1059.

[46] Lian X-Y, Zhang Z, and Stringer JL. (2006) Anticonvulsant and neuroprotective effects of ginsenosides in rats. Epilepsy Res. 70 (2-3): 244-56.

[47] Stringer JL. (2009) Ginseng and other herbal treatments for epilepsy. Encyclopedia of Basic Epilepsy Research. pp. 14451450 .

[48] Khan AW, Khan A, and Ahmed T. (2016) Anticonvulsant, anxiolytic, and sedative activities of Verbena Officinalis. Front Pharmacol. 7: 499.

[49] Perviz S, Khan H, and Pervaiz A. (2016) Plant alkaloids as an emerging therapeutic alternative for the treatment of depression. Front Pharmacol. 7: 28.

[50] Zhu HL, Wan JB, Wang YT, et al. (2013) Medicinal compounds with antiepileptic/anticonvulsant activities. Epilepsia. 55 (1): 12463. 
[51] Peng WH, Lo KL, Lee YH, Hung TH, and Lin YC. (2007) Berberine produces antidepressant-like effects in the forced swim test and in the tail suspension test in mice. Life Sciences. 81 (11): 933-938.

[52] Lambrecq V, Villég F, Marchal C, et al. (2012) Refractory status epilepticus: electroconvulsive therapy as a possible therapeutic strategy. Seizure. 21 (9): 661-4.

[53] Kamel H, Cornes SB, Hegde M, Hall SE, and Josephson SA (2010) Electroconvulsive therapy for refractory status epilepticus: a case series. Neurocritical Care. 12 (2): 204-210.

[54] Ben-Menachem E. (2002) Vagus-nerve stimulation for the treatment of epilepsy The Lancet Neurology. 1 (8): 477-482.

[55] Grimonprez A, Raedt R, Baeken C, Boon P, and Vonck K. (2015) The antidepressant mechanism of action of vagus nerve stimulation: evidence from preclinical studies. Neuroscience and Biobehavioral Reviews. 56: 26-34.

[56] Laxpati NG, Kasoff WS, and Gross RE. (2014) Deep brain stimulation for the treatment of epilepsy: circuits, targets, and trials. Neurotherapeutics. 11 (3): 508-526.

[57] Holtzheimer PE and Mayberg HS. (2011) Deep brain stimulation for psychiatric disorders. Annu Rev Neurosci. 34: 289-307.

[58] Gillig P, Sackellares JC, and Greenberg HS. (1988) Right hemisphere partial complex seizures: mania, hallucinations, and speech disturbances during ictal events. Epilepsia. 29: 2629.

[59] Kaplan PW. (2003) Delirium and epilepsy. Dialogues Clin Neurosci. 5 (2): 187-200.

[60] Beletsky V and Mirsattari SM. (2011) Epilepsy, mental health disorder, or both? Epilepsy Research and Treatment. (Article ID 163731).

[61] Matsuura M, Adachi N, Oana Y, et al. (2004) A polydiagnostic and dimensional comparison of epileptic psychoses and schizophrenia spectrum disorders. Schizophrenia Research. 69 (2-3): 189-201.

[62] Lado FA and Moshé SL. (2008) How do seizures stop? Epilepsia. 49 (10): 1651-54.

[63] Wharton W, Gleason CE, and Asthana S. (2012) Neurobiological underpinnings of the estrogen - mood relationship. Curr Psychiatry Reviews. 8 (3): 247-256. (p. 4).

[64] Huo L, Straub RE, Schmidt PJ, et al. (2007) Risk for premenstrual dysphoric disorder is associated with genetic variation in ESR1, the estrogen receptor alpha gene. Biological Psychiatry. 62 (8): 925-933.

[65] Ting JT and Feng G. (2011) Neurobiology of obsessivecompulsive disorder: insights into neural circuitry dysfunction through mouse genetics. Curr Opin Neurobiol. 21 (6): 842848 .

[66] Lindgren KA, Larson CL, Schaefer SM, et al. (1999) Thalamic metabolic rate predicts EEG alpha power in healthy control subjects but not in depressed patients. Biological Psychiatry. Vol. 45: 943-952.

[67] Plana-Ripoll O, Pedersen CB, and McGrath JJ. (2019) Exploring comorbidity within mental disorders among a Danish national population. JAMA Psychiatry. 76 (3): 259270.
[68] Cassidy CM, Balsam PE, Weinstein JJ, et al. (2018) A perceptual inference mechanism for hallucinations linked to striatal dopamine. Current Biology. 28 (4): 503-514. e4.

[69] Gittelman JX, Perke DJ, and Portfors CV. (2013) Dopamine modulates auditory responses in the inferior colliculus in a heterogeneous manner. J Assoc Res Otolaryngol. 14 (5): 71929.

[70] Hargreave E. (2006). The neuroplasticity phenomenon of kindling. $\mathrm{http}: / /$ hargreaves.swong.webfactional.com/kindle.htm. (Accessed 5/19/18).

[71] Begh M, Beghi E, and Cornaggia CM. (2016) Epilepsy in psychiatric disorders. In: Mula M. (eds) Neuropsychiatric Symptoms of Epilepsy. Neuropsychiatric symptoms of neurological disease. pp. 289-302. Springer, Cham Publishing Company. ISBN: 978-3-319-22158-8.

[72] Josephson CB, Lowerison M, Vallerand I, et al. (2017). Association of depression and treated depression with epilepsy and seizure outcomes: a multicohort analysis. JAMA Neurol. 74 (5): 533-539.

[73] Lopez AY, Wang X, Xu M, et al. (2017) Ankyrin-G isoform imbalance and interneuronopathy link epilepsy and bipolar disorder. Molecular Psychiatry. 22: 1464-1472.

[74] Pearce JMS. (2001) Bromide, the first effective antiepileptic agent. Journal of Neurology, Neurosurgery \& Psychiatry. 72 (3).

[75] Substance Abuse and Mental Health Services Administration, Office of Applied Studies, Rockville, MD. (2008) The NSDUH Report: Substance use and dependence following initiation of alcohol or illicit drug use.

[76] Saisan J, Smith M, Robinson L, and Segal J. (2018) Substance abuse and mental health issues: dealing with drug or alcohol addiction and co-occurring mental health problems. https://www.helpguide.org/articles/addictions/substanceabuse-and-mental-health.htm. (Accessed 9/25/18).

[77] Schäfers M and Sorkin L. (2008) Effect of cytokines on neuronal excitability. Neuroscience Letters. 437 (3): 188-193.

[78] Vezzani A and Viviani B. (2015) Neuromodulatory properties of inflammatory cytokines and their impact on neuronal excitability. Neuropharmacology. 96 (Part A): 70-82.

[79] Galic MA, Riazi K, and Pittman QJ. (2012) Cytokines and brain excitability. Frontiers in Neuroendocrinology. 33 (1): 116-125.

[80] Lu S, Peng H, Wang L, et al. (2013) Elevated specific peripheral cytokines found in major depressive disorder patients with childhood trauma exposure: a cytokine antibody array analysis. Compr Psychiatry. 54 (7): 953-61.

[81] Buckley PF and Miller BJ. (2018) Rheumatoid arthritis drugs for schizophrenia? Psychiatric Annals. 48 (5): 232-236.

[82] Boorman E, Romano GF, Russell A, Mondelli V, and Pariante CM. (2015). Are mood and anxiety disorders inflammatory diseases? Psychiatric Annals. 45 (5): 240-248.

[83] Biskin RS and Paris J. (2013) Comorbidities in borderline personality disorder. Psychiatric Times. 30 (1).

[84] Aghajanian GK and Marek GJ. (1997) Serotonin induces excitatory postsynaptic potentials in apical dendrites of neocortical pyramidal cells. Neuropharmacology. 36 (4-5): 589-599. 
[85] Cardamone L, Salzberg MR, Koe AS, et al. (2014) Chronic antidepressant treatment accelerates Kindling epileptogenesis in rats. Neurobiol Dis. 63: 194-200.

[86] Cardamone L, Salzberg MR, and Jones NC (2013) Antidepressant therapy in epilepsy: can treating the comorbidities affect the underlying disorder? Br J Pharmacol. 168 (7): 1531-1554.

[87] Preskorn SH and Fast GA. (1992). Tricyclic antidepressantinduced seizures and plasma drug concentration. J Clin Psychiatry. 53: 160-162.

[88] Pisani F, Spina E, and Oteri G. (1099). Antidepressant drugs and seizure susceptibility: from in vitro data to clinical practice. Epilepsia. 40 (Suppl. 10): S48-S56.

[89] Ripoll LH. (3013) Psychopharmacologic treatment of borderline personality disorder. Dialogues Clin Neurosci. 15 (2): 213-224.

[90] Olabi B and Hall J. (2010) Borderline personality disorder: current drug treatments and future prospects. Ther Adv Chronic Dis. 1 (2): 59-66.

[91] Ripoll LH, Triebwasser J, and Siever LJ. (2011) Evidencebased pharmacotherapy for personality disorders. International Journal of Neuropsychopharmacology. 14 (9): 1257-1288.

[92] Iranmanesh A, Lawson D, Dunn B, and Veldhuis JD. (2011) Glucose ingestion selectively amplifies ACTH and cortisol secretory-burst mass and enhances their joint synchrony in healthy men. J Clin Endocrinol Metab. 96 (9): 2882-2888.

[93] Landsberg L. (1996) Insulin and the sympathetic nervous system in the pathophysiology of hypertension. Blood Press Supply. 1: 25-29.

[94] Milani P, Piu P, Popa T, et al. (2010) Cortisol-induced effects on human cortical excitability. Brain Stimul. 3 (3): 131-139.

[95] Huang C-W, Huang C, Cheng J-T, Tsai J-J, and Wu SN. (2007) Glucose and hippocampal neuronal excitability: role of ATPsensitive potassium channels. Journal of Neuroscience Research. 85 (7): 1468-1477.

[96] Neilson S. (2018) Trying the Ketogenic Diet for mental health. 10 days of eating fat, for my mental health. SCIENCE OF US.https://www.thecut.com/2018 /01/trying-the-ketogenic-dietfor-mental-health.html. (Accessed 6/18/18).

[97] Gedgaudas NT. (2009) Primal body, primal mind. Beyond the Paleo Diet for total health and a longer life. Healing Arts Press, Rochester, Vermont.

[98] Masino SA, Kawamura M Jr, Wasser CD, Pomeroy LT, and Ruskin DN. (2009) Adenosine, Ketogenic Diet and epilepsy: the emerging therapeutic relationship between metabolism and brain activity. Curr. Neuropharmacol. 7 (3): 257- 268.

[99] Aurora SK and Wilkinson F. (2007) The brain is hyperexcitable in migraine. Cephalalgia. 27 (12) 1442-1453.

[100] Pathophysiology of migraine. https://migraine.com/pro/ pathophysiology-of-migraine/ (Accessed 5/21/18).

[101] Fleming KC and Volcheck MM. (2015) Central Sensitization Syndrome and the initial evaluation of a patient with fibromyalgia: a review. Rambam Maimonides Med J. 6 (2): e0020.

[102] Li Q, Han Y, Dy ABC, and Hagerman RJ. (2017) The gut microbiota and autism spectrum disorders. Front Cell Neurosci.
11: 120 .

[103] Masterjohn C. (2008) High cholesterol and heart disease myth or truth? The response-to-injury rabbit never developed atherosclerosis — why not? (Accessed 9/28/18).

[104] Cohen S, Janicki-Deverts D, Doyle WJ, et al. (2012) Chronic stress, glucocorticoid receptor resistance, inflammation, and disease risk. PNAS. 109 (16): 5995-5999.

[105] Powell ND, Sloan EK, Bailey MT, et al. (2013) Social stress up-regulates inflammatory gene expression in the leukocyte transcriptome via $\beta$-adrenergic induction of myelopoiesis. PNAS. 110 (41): 16574-16579.

[106] Friedman HS and Booth-Kewley S. (1987) Personality, Type A behavior, and coronary heart disease: the role of emotional expression. Journal of Personality and Social Psychology. 53 (4): 783-792.

[107] Griner T. (1995) What's really wrong with you? A revolutionary look at how muscles affect your health. Avery Publishing. New York, NY.

[108] Johnstone T, van Reekum CM, Urry HL, Kalin NH, Davidson, RJ. (2007) Failure to regulate: counterproductive recruitment of top-down prefrontal-subcortical circuitry in major depression. J. Neuroscience. 27 (33): 8877-8884.

[109] Leuchter AF, Cook IA, Hunter AM, Cai C, and Horvath S. (2012) Resting-state quantitative electroencephalography reveals increased neurophysiologic connectivity in depression. 7 (2): 1-13. e32508.

[110] Rigucci S, Serafini G, Pompili M, Kotzalidis GD, and Tatarelli R. (2009). Anatomical and functional correlates in major depressive disorder: the contribution of neuroimaging studies. World J Biol Psychiatry. 11 (2 Pt 2): 165-180.

[111] Kimbrell TA, Ketter TA, George MS, et al. (2002) Regional cerebral glucose utilization in patients with a range of severities of unipolar depression. Biological Psychiatry. 51 (3): 237-252.

[112] Crick F. (1984) Function of the thalamic reticular complex: the Searchlight Hypothesis. PNAS. 81: 4586-4590.

[113] Hamilton JP, Etkin A, Furman DJ, et al. (2012) Functional neuroimaging of major depressive disorder: a meta-analysis and new integration of baseline activation and neural response data. Am J Psychiatry. 169 (7): 693-703.

[114] Erlij D, Acosta-García J, Rojas-Márquez M, et al. (2012) Dopamine D4 receptor stimulation in GABAergic projections of the globus pallidus to the reticular thalamic nucleus and the substantia nigra reticulata of the rat decreases locomotor activity. Neuropharmacology. 62 (2): 1111-1118.

[115] THALAMUS: http://zlab.rutgers.edu/modules/teaching/docs/Thalamus/Thalam us\%20Lecture.pdf. (Accessed 5/19/18).

[116] Carlsson A. (1988) The current status of the dopamine hypothesis of schizophrenia. Psychopharmacology. 1: 179-186.

[117] Al-Shargie F, Kiguchi M, Badruddin N, et al. (2016). Mental stress assessment using simultaneous measurement of EEG and fNIRS. Biomed Opt Express. 7 (10): 3882-3898.

[118] Wada JA, Sato M, and Corcoran ME. (1974) Persistent seizure susceptibility and recurrent spontaneous seizures in kindled cats. Epilepsia. 15 (4): 465-478. 
[119] Baliki MN, Geha PY, Apkarian AV, and Chialvo DR. (2008) Beyond feeling: chronic pain hurts the brain, disrupting the Default-Mode network dynamics. Journal of Neuroscience. 28 (6): 1398-1403.

[120] Kang DH, Son JH, and Kim GC. (2010) Neuroimaging studies of chronic pain. Korean J Pain. 23 (3): 159.

[121] Martucci KT, Ng P, and Mackey S. (2014) Neuroimaging chronic pain: what have we learned and where are we going? Future Neurol. 9 (6): 615-626.

[122] Crone EA and Ridderinkhof KR. (2011) The developing brain: from theory to neuroimaging and back. Developmental Cognitive Neuroscience. 1 (2): 101-109.

[123] Ferreira, MAR, O'Donovan MC, and Sklar P. (2008) Collaborative genome-wide association analysis supports a role for ANK3 and CACNA1C in bipolar disorder. Nat Genet. 40 (9): 1056-1058.

[124] Woolf, CJ. (2011) Central sensitization: implications for the diagnosis and treatment of pain. Pain. 153 (3 suppl): S2-15.

[125] ANK3 ankyrin 3 [Homo sapiens (human)] - Gene- NCBI Gene ID: 288. https://www.ncbi.nlm.nih.gov/gene/288. (Accessed 11/1/18).

[126] Yuan A, Yi Z, Wang Q, et al. (2012) ANK3 as a risk gene for schizophrenia: new data in Han Chinese and meta analysis. Am J Med Genet B Neuropsychiatr Genet. 159B (8): 997-1005.

[127] Lopez AY, Wang X, Xu M, et al. (2017) Ankyrin-G isoform imbalance and interneuronopathy link epilepsy and bipolar disorder. Mol Psychiatry. 22 (10): 1464-1472.

[128] Green EK, Grozeva D, Jones I, et al., Wellcome Trust Case Control Consortium, Holmans, PA, Owen, MJ, O'Donovan, MC, and Craddock N. (2010) The bipolar disorder risk allele at CACNA1C also confers risk of recurrent major depression and of schizophrenia. Mol Psychiatry. 15 (10): 1016-1022.

[129] Liu Y, Blackwood DH, Caesar S, et al. (2011) Meta-analysis of genome-wide association data of bipolar disorder and major depressive disorder. Mol Psychiatry. 16 (1).

[130] Iqbal Z, Vandeweyer G, van der Voet M, et al. (2013) Homozygous and heterozygous disruptions of ANK3: at the crossroads of neurodevelopmental and psychiatric disorders. Human Molecular Genetics. 22: 1960-1970.

[131] Bahremand A, Ziai P, Khodadad TK, et al. (2010) Agmatine enhances the anticonvulsant effect of lithium chloride on pentylenetetrazole-induced seizures in mice: Involvement of L-arginine/nitric oxide pathway. Epilepsy \& Behavior. 18 (3): 186-192.

[132] Brown P, Kashiviswanath S, Huynh A, et al. (2016) Lithium therapy in comorbid temporal lobe epilepsy and cycloid psychosis. Oxf Med Case Reports. 2016 (12): omw089.

[133] Shukla S, Mukherjee S, and Decina P. (1988) Lithium in the treatment of bipolar disorders associated with epilepsy: an open study. J Clin Psychopharmacol. 8 (3): 201-204.

[134] Subramanian J, Dye L, and Morozov, A. (2013) Rap1 Signaling Prevents L-Type Calcium Channel-Dependent Neurotransmitter Release. Journal of Neuroscience. 33 (17): 7245.

[135] Santos M, D'Amico D, Spadoni O, et al. (2013) Hippocampal hyperexcitability underlies enhanced fear memories in TgNTRK3, a panic disorder mouse model. Journal of
Neuroscience. 33 (38): 15259-15271.

[136] Contractor A, Klyachko VA, and Portera-Cailliau C. (2015) Altered neuronal and circuit excitability in Fragile $\mathrm{X}$ syndrome. Neuron. 87 (4): 699-715.

[137] O'Brien NL, Way MJ, Kandaswamy R, et al. (2014) The functional GRM3 Kozak sequence variant rs148754219 affects the risk of schizophrenia and alcohol dependence as well as bipolar disorder. Psychiatric Genetics. 24: 277-278.

[138] Schizophrenia Working Group of the Psychiatric Genomics Consortium: Ripke S, Neale BM, and O'Donovan MC. (2014) Biological insights from 108 schizophrenia-associated genetic loci. Nature. 511 (7510): 421-427.

[139] Freedman R, Coon H, Myles-Worsley M, et al. (1997) Linkage of a neurophysiological deficit in schizophrenia to a chromosome 15 locus. PNAS. 94 (2): 587-592.

[140] Pizzarelli R and Cherubini E. (2011) Alterations of GABAergic signaling in autism spectrum disorders. Neural Plast. 1011: 157193.

[141] Ogawa S, Tsuchimine S, and Kunugi H. (2018) Cerebrospinal fluid monoamine metabolite concentrations in depressive disorder: a meta-analysis of historic evidence. Journal of Psychiatric Research. 105: 137-146.

[142] Shepard RD, Langlois LD, Browne CA, et al. (2018) Ketamine reverses lateral habenula neuronal dysfunction and behavioral immobility in the forced swim test following maternal deprivation in late adolescent rats. Front Synaptic Neurosci. 10: 39.

[143] Popovic J and Mestrovic A. (2014) Habenula - the role in depression. Gyrus. 3 (2): 97-99.

[144] Authement ME, Langlois LD, Shepard RD, et al. (2018) A role for corticotropin-releasing factor signaling in the lateral habenula and its modulation by early-life stress. Sci. Signal. 11 (520): 6480.

[145] Aizawa H, Cui W, Tanaka K, and Okamoto H. (2013) Hyperactivation of the habenula as a link between depression and sleep disturbance. Frontiers in Human Neuroscience. 7: 1-6.

[146] Wang R and Aghajanian G. (1977). Physiological evidence for habenula as major link between forebrain and midbrain raphe. Science. 197: 89-91.

[147] Christoph G, Leonzio R, and Wilcox K. (1986). Stimulation of the lateral habenula inhibits dopamine-containing neurons in the substantia nigra and ventral tegmental area of the rat. J Neurosci. 6 (3): 613-619.

[148] Herrick DD, and Sateia MJ. (2016) Insomnia and depression: a reciprocal relationship. Psychiatric Annals. 46 (3): 164-176.

[149] Engström G, Alling C, Blennow K, Regnell G, and TräskmanBendz L. (1999) Reduced cerebrospinal HVA concentrations and HVA/5-HIAA ratios in suicide attempters. Monoamine metabolites in 120 suicide attempters and 47 controls. Eur. Neuropsychopharmacol. 9 (5): 399-405.

[150] Pande AC, Pollack MH, Crockatt J, et al. (2000) Placebocontrolled study of gabapentin treatment of panic disorder. J Clin Psychopharmacol. 20 (4): 467-471.

[151] Pollack MH, Matthews J, and Scott EL (1998) Gabapentin as a potential treatment for anxiety disorders. American Journal of Psychiatry. 155 (7): 992-993. 
[152] Strawn, JR and Geracioti TD Jr (2007) The treatment of generalized anxiety disorder with pregabalin, an atypical anxiolytic. Neuropsychiatr Dis Treat. 3 (2): 237-243.

[153] Baetz M and Bowen RC. (1998) Efficacy of divalproex sodium in patients with panic disorder and mood instability who have not responded to conventional therapy. Can J Psychiatry. 43 (1): 73-77.

[154] Cournoyer J. (1986) Rapid response of a disorder to the addition of lithium carbonate: panic resistant to tricyclic antidepressants. Can J Psychiatry. 31 (4): 335-8.

[155] Pande AC, Davidson JR, Jefferson JW, et al. (1999) Treatment of social phobia with gabapentin: a placebo controlled study. Clin Psychopharmacol. 19 (4): 341-348.

[156] Berlant, J. (2006) Topiramate as a therapy for chronic posttraumatic stress disorder. Psychiatry (Edgmont). 3 (3): 4045 .

[157] Wang HR, Woo YS, and Bahk W-M. (2014) Potential role of anticonvulsants in the treatment of obsessive-compulsive and related disorders. Psychiatry and Clinical Neurosciences. 68: 723-732.

[158] Mason BJ, Quello S, Goodell V, et al. (2014) Gabapentin Treatment for Alcohol Dependence. A Randomized Clinical Trial. JAMA Intern Med. 174 (1): 70-77.

[159] Marks DM, Patkar AA, Masand PS, and Pae C-U. (2009) Does pregabalin have neuropsychotropic effects?: a short perspective. Psychiatry Investig. 6 (2): 55-58.

[160] Hirschfeld R, Lewis L, and Vornik L. (2003) Perceptions and impact of bipolar disorder: how far have we really come? Results of the National Depressive and Manic-Depressive Association 2000 survey of individuals with bipolar disorder. J Clin Psychiatry. 64 (2): 161-174.

[161] Dagani J, Signorini G, Nielssen O, et al. (2016) Meta-Analysis of the Interval between the Onset and Management of Bipolar Disorder. The Canadian Journal of Psychiatry. 64 (4).

[162] Campbell D. (2012) People with bipolar disorder may wait 13 years for diagnosis. The Guardian. https://www.theguardian.com/society/2012/jun/27/bipolardisorder-diagnosis-survey. (Accessed 10/7/18).

[163] Carpenter WT Jr, Buchanan RW, Kirkpatrick B, and Breier AF. (1999) Diazepam treatment of early signs of exacerbation in schizophrenia. Am J Psychiatry. 156 (2): 299-303.
[164] Wassef AA, Dott SG, Harris A, et al. (2000) Randomized, placebo-controlled pilot study of divalproex sodium in the treatment of acute exacerbations of chronic schizophrenia. J Clin Psychopharmacol. 20: 357-361.

[165] Basan A, Kissling W, and Leucht S. (2004) Valproate as an adjunct to antipsychotics for schizophrenia: a systematic review of randomized trials. Schizophr Res. 70: 33-37.

[166] Gallagher RM, Mueller LL, and Freitag FG. (2002) Divalproex sodium in the treatment of migraine and cluster headaches. J Am Osteopath Assoc. 102 (2): 92.

[167] Boomershine CS. (2010) Pregabalin for the management of fibromyalgia syndrome. J Pain Res. 3: 81-88.

[168] Tremont-Lukats and Beckonja MC. (2000) Anticonvulsants for neuropathic pain syndromes: mechanisms of action and place in therapy. Drugs. 60 (5): 1029-1052.

[169] Wood JG, Crager JL, Delap CM, and Heiskell KD. (2007) Beyond methylphenadate: nonstimulant medications for youth with ADHD. J Atten Disord. 11 (3): 341-350.

[170] Eatmon CV and Daniel JS. (2014) Should you use an anticonvulsant to treat impulsivity and aggression? Current Psychiatry. 13 (4): 49-51.

[171] Hillhouse TM and Porter JH. (2015) A brief history of the development of antidepressant drugs: from monoamines to glutamate. Exp Clin Psychopharmacol. 23 (1): 1-21.

[172] Ramachandraih CT, Subramanyam N, Bar KJ, Baker G, and Yeragani VK. (2011) Antidepressants: from MAOIs to SSRIs and more. Indian Journal of Psychiatry. 53 (2): 180-182.

[173] Kreston R. (2016) The psychic energizer!: the serendipitous discovery of the first antidepressant - Body Horrors. $\mathrm{http}: / /$ blogs.discovermagazine.com/bodyhorrors/2016/01/27/2 081/. (Accessed 10/2/18).

[174] Neumeister A, Nugent AC, Waldeck T, et al. (2004) Neural and behavioral responses to tryptophan depletion in unmedicated patients with remitted major depressive disorder and controls. Arch Gen Psychiatry. 61: 765-773.

[175] Delgado PL, Price LH, Miller HL, et al. (1994) Serotonin and the Neurobiology of Depression. Effects of tryptophan depletion in drug-free depressed patients. Arch Gen Psychiatry. 51: 865-874. 